Document downloaded from:

http://hdl.handle.net/10251/139925

This paper must be cited as:

Caraballo, T.; Cortés, J.; Navarro-Quiles, A. (2019). Applying the Random Variable Transformation method to solve a class of random linear differential equation with discrete delay. Applied Mathematics and Computation. 356:198-218.

https://doi.org/10.1016/j.amc.2019.03.048

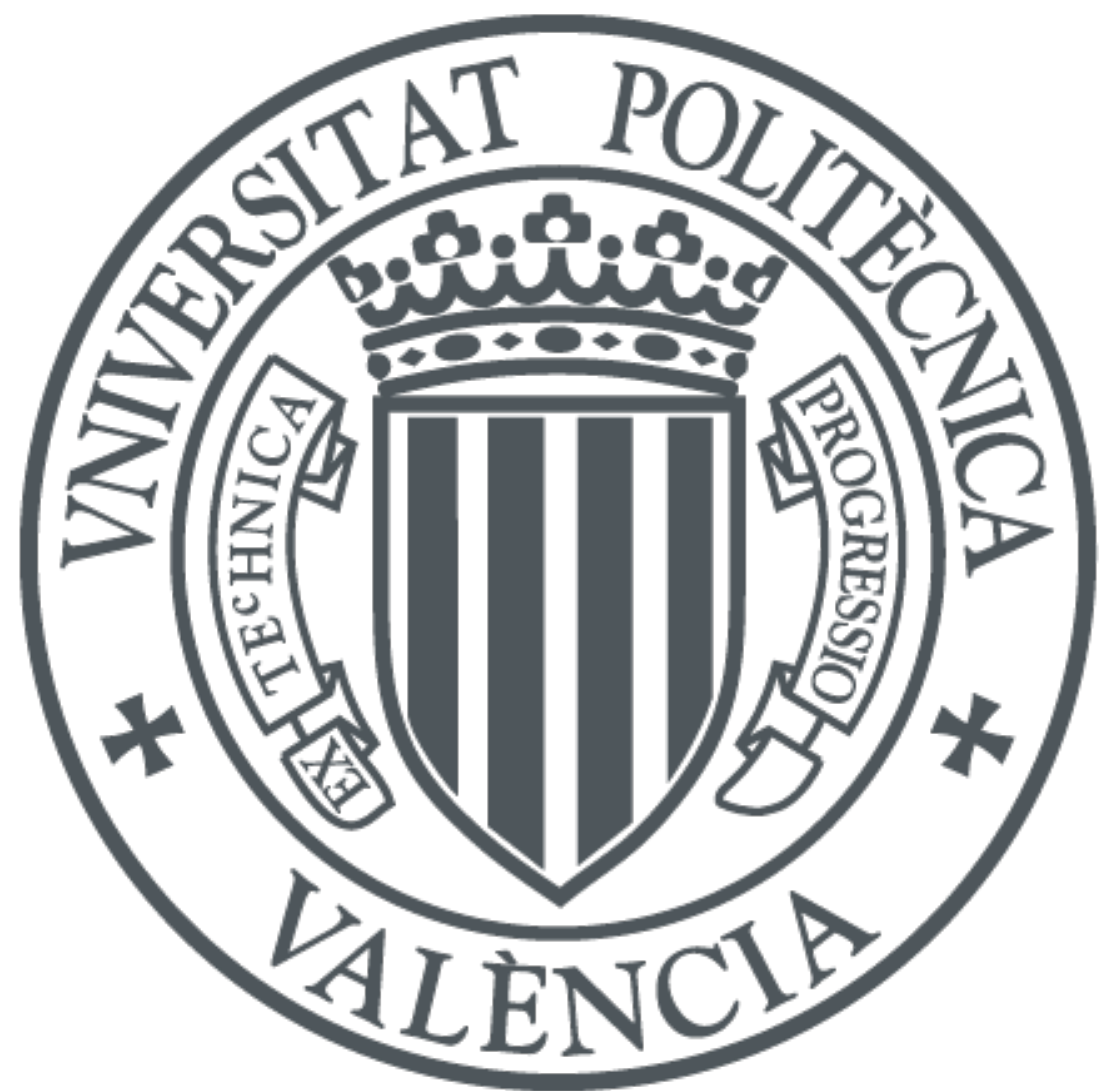

The final publication is available at

https://doi.org/10.1016/j.amc.2019.03.048

Copyright Elsevier

Additional Information 


\title{
Applying the Random Variable Transformation method to solve a class of random linear differential equation with discrete delay
}

\author{
Tomás Caraballo ${ }^{\mathrm{a}}$, J.-C. Cortés ${ }^{\mathrm{b}}$, A. Navarro-Quiles ${ }^{\mathrm{c}, \mathrm{d}, *}$ \\ ${ }^{a}$ Dpto. Ecuaciones Diferenciales y Análisis Numérico, \\ Universidad de Sevilla, \\ c/ Tarfia s/n, 41012, Sevilla, Spain \\ ${ }^{b}$ Instituto Universitario de Matemática Multidisciplinar, \\ Universitat Politècnica de València, \\ c/ Camino de Vera s/n, 46022, Valencia, Spain \\ ${ }^{c}$ DeustoTech, University of Deusto, 48007 Bilbao, \\ Basque Country, Spain \\ ${ }^{d}$ Facultad de Ingenieria, Universidad de Deusto, \\ Avda.Universidades, 24, 48007, Bilbao, Basque Country, Spain.
}

\begin{abstract}
We randomize the following class of linear differential equations with delay, $x_{\tau}^{\prime}(t)=a x_{\tau}(t)+$ $b x_{\tau}(t-\tau), t>0$, and initial condition, $x_{\tau}(t)=g(t),-\tau \leq t \leq 0$, by assuming that coefficients $a$ and $b$ are random variables and the initial condition $g(t)$ is a stochastic process. We consider two cases, depending on the functional form of the stochastic process $g(t)$, and then we solve, from a probabilistic point of view, both random initial value problems by determining explicit expressions to the first probability density function, $f(x, t ; \tau)$, of the corresponding solution stochastic processes. Afterwards, we establish sufficient conditions on the involved random input parameters in order to guarantee that $f(x, t ; \tau)$ converges, as $\tau \rightarrow 0^{+}$, to the first probability density function, say $f(x, t)$, of the corresponding associated random linear problem without delay $(\tau=0)$. The paper concludes with several numerical experiments illustrating our theoretical findings.
\end{abstract}

Keywords: Random linear differential equation with delay, Probability density function, Random Variable Transformation technique.

\section{Introduction and motivation}

Ordinary differential equations are useful mathematical tools to model phenomena in areas like Physics, Engineering, Epidemiology, Economics, etc. In many applications, differential equations are formulated using the principle of causality based upon the fact that future state of a system under study is independent of its past state and is solely determined by the current state. Although many phenomena can be properly described using this tenet, there are other situations

\footnotetext{
${ }^{*}$ Corresponding author

Email addresses: carabalı@us.es (Tomás Caraballo), jccortes@imm.upv.es (J.-C. Cortés), annaqui@doctor.upv.es (A. Navarro-Quiles )
} 
7 where it may be more realistic to model the current state of a physical system (understood in 8 a wide sense) in terms of past information. In that case, it is more suitable to describe the 9 dynamics of the physical system by means of differential equations incorporating the past history 1 of the system under analysis. These kinds of differential equations are usually referred to as 11 delay differential equations (DDEs). One commonly distinguishes two main classes of DDEs 12 depending on the type of delay considered therein. If only a part of the history has a relevant 13 influence on the current state, then discrete DDEs are formulated, while continuous DDEs are 14 those whose delay is unbounded or infinite. In this latter case, the whole past history is taken into 15 account to describe the phenomenon under study. In this paper, we will deal with the following 16 class of linear discrete DDEs with initial condition

$$
\left\{\begin{array}{l}
x_{\tau}^{\prime}(t)=a x_{\tau}(t)+b x_{\tau}(t-\tau), \quad t>0, \quad \tau>0, \\
x_{\tau}(t)=g(t), \quad-\tau \leq t \leq 0,
\end{array}\right.
$$

where $\tau>0$ denotes a prefixed finite delay, $a$ is the coefficient of the non-delay term, $x_{\tau}(t), b$ is the coefficient of the delay term, $x_{\tau}(t-\tau)$, and $g(t)$ is an arbitrary function (initial condition) defined on the interval $[-\tau, 0]$.To avoid confusion with the notation introduced for the initial condition and the solution of the initial value problem (IVP) formulated in (1), hereinafter the solution will be denoted by $x_{\tau}(\cdot)$. This notation is necessary to explicitly indicate the dependence of the solution on the $\tau$ parameter since later we will study the convergence of the solution of the IVP (1) as $\tau \rightarrow 0^{+}$to the solution of the corresponding IVP without delay. Now, if we assume that $g(t)$ is continuous in $[-\tau, 0]$ and differentiable in $]-\tau, 0[$ then, according to Theorem 1 below, there exists an exact expression of the solution of IVP (1).

Theorem $1([1,2])$. Let us consider IVP (1) and assume that $g(t)$ is continuous in $[-\tau, 0]$ and differentiable in $]-\tau, 0\left[\right.$, i.e., $g(\cdot) \in C^{1}([-\tau, 0])$. Then, IVP $(1)$ has a unique solution $x_{\tau}(\cdot) \in$ $C^{0}([-\tau, \infty]) \cap C^{1}([-\tau, 0]) \cap C^{1}([0, \infty])$ given by

$$
x_{\tau}(t)=\mathrm{e}^{a(t+\tau)} \mathrm{e}_{\tau}^{b_{1}, t} g(-\tau)+\int_{-\tau}^{0} \mathrm{e}^{a(t-s)} \mathrm{e}_{\tau}^{b_{1}, t-\tau-s}\left(g^{\prime}(s)-a g(s)\right) \mathrm{d} s,
$$

where $b_{1}=\mathrm{e}^{-a \tau} b$, and $\mathrm{e}_{\tau}^{b_{1}, t}$ and $\mathrm{e}_{\tau}^{b_{1}, t-\tau-s}$ denote the delayed exponential function, $\mathrm{e}_{\tau}^{c, t}$, evaluated at $(c, t)=\left(b_{1}, t\right)$ and $(c, t)=\left(b_{1}, t-\tau-s\right)$, respectively. This function appears in a natural way in dealing with the linear discrete DDE (1) since its solution is constructed segment by segment (see for example [2]). For the sake of completeness, below we recall its definition.

Definition 1. ([2]) Let $c$ be a real number and $\tau>0$, then the function

$$
\mathrm{e}_{\tau}^{c, t}=\left\{\begin{array}{cc}
0, & -\infty<t<-\tau, \\
1, & -\tau \leq t<0, \\
1+c \frac{t}{1 !}, & 0 \leq t<\tau, \\
1+c \frac{t}{1 !}+c^{2} \frac{(t-\tau)^{2}}{2 !}, & \tau \leq t<2 \tau, \\
\vdots & \vdots \\
\sum_{k=0}^{n} c^{k} \frac{(t-(k-1) \tau)^{k}}{k !}, & (n-1) \tau \leq t<n \tau,
\end{array}\right.
$$

${ }_{34}$ is called the delayed exponential function, where $n=\lfloor t / \tau\rfloor+1$, being $\lfloor x\rfloor$ the greatest integer less 35 than or equal to $x$. 
So far we have revised the main definitions and results involving the solution of deterministic IVP (1) for the linear discrete DDE. When this class of equations is applied to model the dynamics of real phenomena, its input parameters, i.e. the coefficients $a$ and $b$, and the initial condition, $g(t)$, must be fixed from experimental data which often involve uncertainties because they are obtained after measurements and sampling. This fact allows us to treat the input coefficients, $a$ and $b$, as random variables (RVs), and the initial condition, $g(t)$, as a stochastic process (SP) rather than deterministic constants and a classical function, respectively. This leads to the full randomization of IVP (1)

$$
\left\{\begin{array}{l}
x_{\tau}^{\prime}(t ; \omega)=a(\omega) x_{\tau}(t ; \omega)+b(\omega) x_{\tau}(t-\tau ; \omega), \quad t>0, \quad \tau>0 \\
x_{\tau}(t ; \omega)=g(t ; \omega), \quad-\tau \leq t \leq 0,
\end{array}\right.
$$

where $a(\omega)$ and $b(\omega)$ are assumed to be absolutely continuous RVs and $g(t ; \omega)$ is a SP, being all of them defined on a common complete probability space $(\Omega, \mathcal{F}, \mathbb{P})$. In accordance with Theorem 1, and using the so-called "sample random calculus" for SPs [3, App. I], hereinafter it will be assumed that the initial condition SP, $g(t ; \omega)$, satisfies the following condition,

$$
g(\cdot ; \omega) \in C^{1}([-\tau, 0] \times \Omega), \quad \text { a.e. },
$$

where, as usual, a.e. stands for 'almost everywhere', so that the existence of a unique sample solution SP, $x_{\tau}(t ; \omega)$, to random IVP (4) can be guaranteed.

The study of differential equations with delay involving uncertainties has been studied from different approaches. In [4], authors study a class of stochastic impulsive differential equations involving Bernoulli distribution where trial lengths vary randomly. In [5], the complete controllability property of a class of nonlinear stochastic differential equation with delay, in the fractional sense, is investigated assuming that delays are described by Poisson jumps. Stochastic differential equations with delay have been proposed to model interesting real problems. For example, in [6] the nonlinear delay differential neoclassical growth model is analysed assuming that stochastic perturbations of the white noise type. In [7], authors provide sufficient conditions for stability in probability of the equilibrium point of a social obesity epidemic model with distributed delay and stochastic perturbations. In dealing with delay differential equations it is usual to study the behaviour of the solution when the delay tends to zero, i.e., to investigate conditions under which there is convergence of the solution of the IVP with delay to the corresponding solution of the IVP when the delay vanishes. In [8] it is shown that the solution of a mixed stochastic delay differential equation depends continuously on the coefficients and the initial data. In addition, authors prove the convergence of the solutions to equations with vanishing delay to the solution of corresponding equations without delay. In [9] one deals with the mean square convergence and mean square exponential stability of an Euler scheme for a linear impulsive stochastic delay differential equation.

Solving a random (ordinary/partial/fractional/delay/etc.) differential equation means not just to obtain an exact/approximate solution SP, but also its main statistical properties, like the mean and the variance functions. These equations are said to be solved, from a probabilistic standpoint, when the first probability density function (1-PDF) of the solution SP is exact/approximately obtained since from this deterministic function one can completely characterize the probabilistic behaviour of the solution SP at every time instant. As a consequence, mean, variance, skewness, etc., as well as any one-dimensional moment of the solution SP can be derived from the 1-PDF, provided these moments exist. To be specific, if $f(x, t ; \tau)$ denotes the 1-PDF of the solution SP $x_{\tau}(t ; \omega)$ to random IVP $(1)$, then the mean, $\mathbb{E}\left[x_{\tau}(t ; \omega)\right]$, and the variance, $\mathbb{V}\left[x_{\tau}(t ; \omega)\right]$, functions 
77 can be obtained by

$$
\mathbb{E}\left[\left(x_{\tau}(t ; \omega)\right)^{k}\right]=\int_{\mathbb{R}} x^{k} f(x, t ; \tau) \mathrm{d} x, \quad k=1,2, \ldots
$$

78 as

$$
\mathbb{E}\left[x_{\tau}(t ; \omega)\right]=\int_{\mathbb{R}} x f(x, t ; \tau) \mathrm{d} x, \quad \mathbb{V}\left[x_{\tau}(t ; \omega)\right]=\int_{\mathbb{R}} x^{2} f(x, t ; \tau) \mathrm{d} x-\left(\mathbb{E}\left[x_{\tau}(t ; \omega)\right]\right)^{2},
$$

respectively. Fixed a time instant, say $\hat{t}$, the computation of the probability that the solution, $x_{\tau}(\hat{t} ; \omega)$, lies within an interval of specific interest can also be computed just by integrating the $1-\mathrm{PDF}$

$$
\mathbb{P}\left[\omega \in \Omega: a \leq x_{\tau}(\hat{t} ; \omega) \leq b\right]=\int_{a}^{b} f(x, \hat{t} ; \tau) \mathrm{d} x .
$$

At this point is important to stress that, to the best of our knowledge, this approach has already been dealt with some classes of random fractional, ordinary and partial differential equations and of random difference equations as well (see for instance, [10], [11, 12, 13, 14, 15], [16, 17, 18, 19] and [20, 21], respectively), but the corresponding analysis for random DDEs has not been addressed yet.

In the spirit of these previous contributions, the main objective of this paper is solving, from a probabilistic point of view, random IVP (4) by obtaining the 1-PDF, $f(x, t ; \tau)$, of its solution SP, $x_{\tau}(t ; \omega)$, which, according to the deterministic solution formulated in (2), for each $t \in[(n-$ 1) $\tau, n \tau[$, with $\tau>0$ fixed and $n=1,2, \ldots$, is given by

$$
x_{\tau}(t ; \omega)=\mathrm{e}^{a(\omega)(t+\tau)} \mathrm{e}_{\tau}^{b_{1}(\omega), t} g(-\tau ; \omega)+\int_{-\tau}^{0} \mathrm{e}^{a(\omega)(t-s)} \mathrm{e}_{\tau}^{b_{1}(\omega), t-\tau-s}\left(g^{\prime}(s ; \omega)-a(\omega) g(s ; \omega)\right) \mathrm{d} s,
$$

where $b_{1}(\omega)=\mathrm{e}^{-a(\omega) \tau} b(\omega)$. The key tool that will be applied to achieve this goal is the Random Variable Transformation (RVT) method. This technique allows us to obtain the PDF of a random vector, which results from mapping another random vector whose PDF is known. The following result provides the RVT technique in its multidimensional version.

Theorem 2 (Multidimensional RVT method, [3]). Let $\mathbf{x}(\omega)=\left(x_{1}(\omega), \ldots, x_{m}(\omega)\right)$ and $\mathbf{y}(\omega)=$ $\left(y_{1}(\omega), \ldots, y_{m}(\omega)\right)$ be two m-dimensional absolutely continuous random vectors defined on a complete probability space $(\Omega, \mathcal{F}, \mathbb{P})$. Let $\mathbf{r}: \mathbb{R}^{m} \rightarrow \mathbb{R}^{m}$ be a one-to-one deterministic transformation of $\mathbf{x}(\omega)$ into $\mathbf{y}(\omega)$, i.e., $\mathbf{y}(\omega)=\mathbf{r}(\mathbf{x}(\omega))$. Assume that $\mathbf{r}$ is continuous in $\mathbf{x}$ and has continuous partial derivatives with respect to $\mathbf{x}$. Then, if $f_{\mathbf{x}}(\mathbf{x})$ denotes the joint probability density function of the random vector $\mathbf{x}(\omega)$, and $\mathbf{s}=\mathbf{r}^{-1}=\left(s_{1}\left(y_{1}, \ldots, y_{m}\right), \ldots, s_{m}\left(y_{1}, \ldots, y_{m}\right)\right)$ represents the inverse mapping of $\mathbf{r}=\left(r_{1}\left(x_{1}, \ldots, x_{m}\right), \ldots, r_{m}\left(x_{1}, \ldots, x_{m}\right)\right)$, the joint probability density function of the random vector $\mathbf{y}(\omega)$ is given by

$$
g_{\mathbf{y}}(\mathbf{y})=f_{\mathbf{x}}(\mathbf{s}(\mathbf{y}))\left|J_{m}\right|,
$$

where $\left|J_{m}\right|$, which is assumed to be different from zero, denotes the absolute value of the Jacobian defined by the determinant

$$
J_{m}=\operatorname{det}\left(\begin{array}{ccc}
\frac{\partial s_{1}\left(y_{1}, \ldots, y_{m}\right)}{\partial y_{1}} & \ldots & \frac{\partial s_{m}\left(y_{1}, \ldots, y_{m}\right)}{\partial y_{1}} \\
\vdots & \ddots & \vdots \\
\frac{\partial s_{1}\left(y_{1}, \ldots, y_{m}\right)}{\partial y_{m}} & \cdots & \frac{\partial s_{m}\left(y_{1}, \ldots, y_{m}\right)}{\partial y_{m}}
\end{array}\right)
$$


Once we have obtained the 1-PDF, $f(x, t ; \tau)$, of the solution SP to random IVP (4), the next objective of this paper is to study the relationship between $f(x, t ; \tau)$ and the 1-PDF, say $f(x, t)$, of the corresponding random IVP without delay, i.e.,

$$
\left\{\begin{aligned}
x^{\prime}(t ; \omega) & =(a(\omega)+b(\omega)) x(t ; \omega), \quad t>0, \\
x(0 ; \omega) & =g(0 ; \omega)=g_{0}(\omega),
\end{aligned}\right.
$$

where $g_{0}(\omega)$ is an absolutely continuous RV. To be specific, we will establish conditions in order to guarantee that

$$
\lim _{\tau \rightarrow 0^{+}} f(x, t ; \tau)=f(x, t), \quad \text { for each }(x, t) \in \mathcal{D}\left(x_{\tau}(t ; \omega)\right) \cap \mathcal{D}(x(t ; \omega)) \times[(n-1) \tau, n \tau[\text { fixed }
$$

being $n=1,2, \ldots$ and where $\mathcal{D}\left(x_{\tau}(t ; \omega)\right)$ and $\mathcal{D}(x(t ; \omega))$ denote the codomains of SPs $x_{\tau}(t ; \omega)$ and $x(t ; \omega)$, respectively. This analysis will focus on the following choices of the initial condition $g(t ; \omega)$ for random IVP (4)

- Case I: $g(t ; \omega)=\mathrm{e}^{a(\omega) t+c(\omega)}$.

- Case II: $g(t ; \omega)=\sum_{j=0}^{m} c_{j}(\omega) t^{j}, m \geq 0$.

The choice in Case I has been made because, as it will be seen later, it allows us to deal with a scenario that illustrates adequately the main ideas of our approach. While Case II involves the general case in which the initial condition is a random polynomial of degree $m$, which has interest by itself since, similarly to what happens in the deterministic context, many important random SPs can be approximated by appropriate random polynomials.

The rest of this paper is organized as follows. Section 2 is split into two subsections, in the first one, we determine an explicit expression to the 1-PDF, $f(x, t ; \tau)$, of the solution SP to random IVP (1) in Case I, i.e., when $g(t ; \omega)=\mathrm{e}^{a(\omega) t+c(\omega)}$. Subsection 2.1 is addressed to establish sufficient conditions upon the involved random input parameters $a(\omega), b(\omega)$ and $c(\omega)$, in order to guarantee that $f(x, t ; \tau)$ converges, as $\tau \rightarrow 0^{+}$, to the 1-PDF, $f(x, t)$, of the corresponding associated random linear problem without delay $(\tau=0)$. For the sake of clarity, Section 3 is organized analogously as Section 2 to conduct the corresponding study in Case II, i.e., when the initial condition to random IVP (1) is a random polynomial $g(t ; \omega)=\sum_{j=0}^{m} c_{j}(\omega) t^{j}, m \geq$ 0 . Several numerical examples, corresponding to Cases I and II are exhibited in Section 4. Conclusions are drawn in Section 5.

\section{Case I: Computing the 1-PDF of the solution SP and study of the convergence}

Let us consider random IVP (4) where the initial condition is given by the SP $g(t ; \omega)=$ $\mathrm{e}^{a(\omega) t+c(\omega)}$. Observe that this situation corresponds to the case where $g(t ; \omega)$ is the solution of the following random IVP

$$
\left\{\begin{array}{l}
g^{\prime}(t ; \omega)=a(\omega) g(t ; \omega), \quad t \geq-\tau, \quad \tau>0 \\
g(-\tau ; \omega)=\mathrm{e}^{-a(\omega) \tau+c(\omega)} .
\end{array}\right.
$$

In other words, we are then implicitly considering a stochastic control problem defined by (4) and (10). In agreement with (7), the solution SP of this control problem is given by

$$
x_{\tau}(t ; \omega)=\mathrm{e}^{a(\omega) t+c(\omega)} \mathrm{e}_{\tau}^{b_{1}(\omega), t}, \quad t \in[(n-1) \tau, n \tau[, \quad n \in \mathbb{N} \text { fixed },
$$


where $b_{1}(\omega)=\mathrm{e}^{-a(\omega) \tau} b(\omega)$. Henceforth, we will assume that the random inputs $a(\omega), b(\omega)$ and $c(\omega)$ are absolutely continuous dependent RVs with a joint PDF denoted by $f_{c, a, b}(c, a, b)$, which is assumed to be known. Observe that $g(t ; \omega)$ satisfies condition (5).

\subsection{Computing the PDF}

First at all, notice that if $\hat{t}$ is such that $x_{\tau}(\hat{t} ; \omega)=0$, then clearly the 1-PDF is given by $f(x, \hat{t} ; \tau)=\delta(x),-\infty<x<\infty$, being $\delta(\cdot)$ the Dirac delta function. Thus, in order to determine the 1-PDF of the solution SP, $x_{\tau}(t ; \omega)$, given by (11), we will only consider time instants $t$ such that $x_{\tau}(t ; \omega) \neq 0$ a.e. As a consequence, as $x_{\tau}(t ; \omega)=\mathrm{e}^{a(\omega) t+c(\omega)} \mathrm{e}_{\tau}^{b_{1}(\omega), t}$, one derives that $\mathrm{e}_{\tau}^{b_{1}(\omega), t} \neq 0$ a.e., at every time instant $t$ where the 1-PDF $f(x, t ; \tau)$ is going to be determined.

Let $t \in[(n-1) \tau, n \tau$ [ be fixed, next we will apply the RVT method (see Theorem 2), in order to obtain the PDF, $f(x, t ; \tau)$, of the solution SP, $x_{\tau}(t ; \omega)$, given by (11). This PDF will be expressed in terms of the joint $\operatorname{PDF} f_{c, a, b}(c, a, b)$. To this end, consider the following deterministic mapping $\mathbf{r}: \mathbb{R}^{3} \longrightarrow \mathbb{R}^{3}$

$$
\begin{aligned}
& x_{1}=r_{1}(c, a, b)=\mathrm{e}^{a t+c} \mathrm{e}_{\tau}^{b_{1}, t}, \\
& x_{2}=r_{2}(c, a, b)=a, \\
& x_{3}=r_{3}(c, a, b)=b,
\end{aligned}
$$

39 $\quad$ where $b_{1}=\mathrm{e}^{-a \tau} b$. The inverse mapping, $\mathbf{s}: \mathbb{R}^{3} \longrightarrow \mathbb{R}^{3}$, of $\mathbf{r}$ is given by

$$
\begin{aligned}
& c=s_{1}\left(x_{1}, x_{2}, x_{3}\right)=\ln \left(\frac{x_{1} \mathrm{e}^{-x_{2} t}}{\mathrm{e}_{\tau}^{b_{1}, t}}\right), \\
& a=s_{2}\left(x_{1}, x_{2}, x_{3}\right)=x_{2}, \\
& b=s_{3}\left(x_{1}, x_{2}, x_{3}\right)=x_{3},
\end{aligned}
$$

where $b_{1}=\mathrm{e}^{-x_{2} \tau} x_{3}$. Observe that the Jacobian of $\mathbf{s}$ is $\left|J_{3}\right|=1 /\left|x_{1}\right| \neq 0$. Moreover, $\left|J_{3}\right|$ is welldefined since $x_{\tau}(t ; \omega)=x_{1} \neq 0$ a.s. for each instant time $t$. Therefore, applying the RVT method one obtains the PDF of the random vector $\left(x_{1}(\omega), x_{2}(\omega), x_{3}(\omega)\right)$,

$$
f\left(x_{1}, x_{2}, x_{3}\right)=f_{c, a, b}\left(\ln \left(\frac{x_{1} \mathrm{e}^{-x_{2} t}}{\mathrm{e}_{\tau}^{b_{1}, t}}\right), x_{2}, x_{3}\right) \frac{1}{\left|x_{1}\right|} \text {, where } b_{1}=\mathrm{e}^{-x_{2} \tau} x_{3} .
$$

Then, marginalizing with respect to the random vector $\left(x_{2}(\omega), x_{3}(\omega)\right)=(a(\omega), b(\omega))$ and taking $t \in\left[(n-1) \tau, n \tau\left[\right.\right.$ arbitrary, one gets the 1-PDF of the solution SP, $x_{\tau}(t ; \omega)$,

$$
f(x, t ; \tau)=\int_{\mathbb{R}^{2}} f_{c, a, b}\left(\ln \left(\frac{x \mathrm{e}^{-a t}}{\mathrm{e}_{\tau}^{b_{1}, t}}\right), a, b\right) \frac{1}{|x|} \mathrm{d} a \mathrm{~d} b, \text { where } b_{1}=\mathrm{e}^{-a \tau} b .
$$

\subsection{Convergence}

As it has been indicated previously, this subsection is devoted to investigate the relationship between the 1-PDF, $f(x, t ; \tau)$, given in (12), as $\tau \rightarrow 0^{+}$, and the 1-PDF, $f(x, t)$, of the solution SP to random IPV (8). In order for the corresponding analysis makes sense (put $\tau \rightarrow 0^{+}$in the initial condition of (10)), we will take as initial condition in this latter IVP $g_{0}(\omega)=\mathrm{e}^{c(\omega)}$, so the solution SP to random IPV (8) is given by

$$
x(t ; \omega)=\mathrm{e}^{c(\omega)} \mathrm{e}^{(a(\omega)+b(\omega)) t} .
$$


By applying the RVT method, it can be checked that the 1-PDF of $x(t ; \omega)$ is given by

$$
f(x, t)=\int_{\mathbb{R}^{2}} f_{c, a, b}\left(\ln \left(x \mathrm{e}^{-(a+b) t}\right), a, b\right) \frac{1}{|x|} \mathrm{d} a \mathrm{~d} b .
$$

152

In order to find out sufficient conditions that guarantee the convergence given in (9), where $f(x, t ; \tau)$ and $f(x, t)$ are given by (12) and (13), respectively, we first establish the following result that permits relating the delayed exponential function to the (classical) exponential function as the delay tends to zero. The proof of this result is based upon the ideas exhibited in [22, Theorem A.3.].

Theorem 3 (Convergence of the delayed exponential function). Let $c \in \mathbb{R}, \tau_{0}>0, \alpha=1+$ $\mathrm{e}^{\tau_{0}|c|}>1$. Then, for any $\left.\tau \in\right] 0, \tau_{0}[$,

$$
\left|\mathrm{e}^{c t}-\mathrm{e}_{\tau}^{c, t}\right| \leq \tau|c|\left(\mathrm{e}^{\alpha|c| T}+\mathrm{e}^{|c|(T-\tau)}\right), \quad t \in[0, T] .
$$

If $\tau=0$ then $\mathrm{e}^{c t}=\mathrm{e}_{\tau}^{c, t}$.

Proof Let $\tau \in] 0, \tau_{0}[$. We will apply mathematical induction in order to prove that for any $n \in \mathbb{N}$

$$
\left|\mathrm{e}^{c t}-\mathrm{e}_{\tau}^{c, t}\right| \leq \tau|c|\left(\mathrm{e}^{\alpha|c| n \tau}+\mathrm{e}^{|c|(n-1) \tau}\right), \quad \text { for } t \in[(n-1) \tau, n \tau] .
$$

- If $n=1$, then $t \in[0, \tau]$ and, by the definition of the delayed exponential function given in (3), $\mathrm{e}_{\tau}^{c, t}=1+c t$. Therefore, we must prove

$$
\left|\mathrm{e}^{c t}-(1+c t)\right| \leq \tau|c|\left(\mathrm{e}^{\alpha|c| \tau}+1\right)
$$

Applying the Fundamental Calculus Theorem (FCT) and the Mean Value Theorem (MVT) for integration, one derives

$$
\begin{aligned}
& \left|\mathrm{e}^{c t}-(1+c t)\right| \leq\left|\mathrm{e}^{c t}-1\right|+|c t| \stackrel{\mathrm{FCT}}{=}\left|\int_{0}^{t}\left[\frac{\mathrm{d}}{\mathrm{d} x}\left(\mathrm{e}^{c x}\right)\right] \mathrm{d} x\right|+|c| t \leq \int_{0}^{t}\left|\frac{\mathrm{d}}{\mathrm{d} x}\left(\mathrm{e}^{c x}\right)\right| \mathrm{d} x+|c| t \\
& =|c| \int_{0}^{t} \mathrm{e}^{c x} \mathrm{~d} x+|c| t \stackrel{t \leq \tau}{\leq}|c| \int_{0}^{\tau} \mathrm{e}^{c x} \mathrm{~d} x+|c| \tau \stackrel{\text { MVT }}{\leq}|c| \tau \mathrm{e}^{c \delta}+|c| \tau \stackrel{\text { (I) }}{\leq}|c| \tau\left(\mathrm{e}^{\alpha|c| \tau}+1\right) .
\end{aligned}
$$

Now, we justify Step (I) previously applied.

Step (I): By the MVT, $\delta \in[0, \tau]$. Let $\alpha>1$, then

$$
c \delta \leq|c| \delta \leq|c| \tau \leq \alpha|c| \tau,
$$

and, as the exponential is an increasing function, $\mathrm{e}^{c \delta} \leq \mathrm{e}^{\alpha|c| \tau}$.

- Now, assuming that claim (14) is satisfied for $n \geq 1$ (induction hypothesis), we will apply the FCT to prove inequality (14) for $n+1$. Let $t \in[n \tau,(n+1) \tau]$, 


$$
\begin{aligned}
& \left|\mathrm{e}_{\tau}^{c, t}-\mathrm{e}^{c t}\right| \stackrel{\text { (II) }}{\leq}|c| \tau\left(\mathrm{e}^{\alpha|c| n \tau}+\mathrm{e}^{|c|(n-1) \tau}\right)+\int_{n \tau}^{(n+1) \tau}\left|\frac{\mathrm{d}}{\mathrm{d} s}\left(\mathrm{e}_{\tau}^{c, s}-\mathrm{e}^{c s}\right)\right| \mathrm{d} s \\
& =|c| \tau\left(\mathrm{e}^{\alpha|c| n \tau}+\mathrm{e}^{|c|(n-1) \tau}\right)+|c| \int_{n \tau}^{(n+1) \tau}\left|\mathrm{e}_{\tau}^{c, s-\tau}-\mathrm{e}^{c s}\right| \mathrm{d} s \\
& \leq|c| \tau\left(\mathrm{e}^{\alpha|c| n \tau}+\mathrm{e}^{|c|(n-1) \tau}\right)+|c| \int_{n \tau}^{(n+1) \tau}\left|\mathrm{e}_{\tau}^{c, s-\tau}-\mathrm{e}^{c(s-\tau)}\right| \mathrm{d} s \\
& +|c| \int_{n \tau}^{(n+1) \tau}\left|\mathrm{e}^{c(s-\tau)}-\mathrm{e}^{c s}\right| \mathrm{d} s \\
& \stackrel{\text { (III) }}{\leq}|c| \tau\left(\mathrm{e}^{\alpha|c| n \tau}+\mathrm{e}^{|c|(n-1) \tau}\right)+|c| \int_{n \tau}^{(n+1) \tau}|c| \tau\left(\mathrm{e}^{\alpha|c| n \tau}+\mathrm{e}^{|c|(n-1) \tau}\right) \mathrm{d} s \\
& +|c| \int_{n \tau}^{(n+1) \tau} \int_{s-\tau}^{s}\left|\frac{\mathrm{d}}{\mathrm{d} \sigma}\left(\mathrm{e}^{c \sigma}\right)\right| \mathrm{d} \sigma \mathrm{d} s \\
& =|c| \tau\left(\mathrm{e}^{\alpha|c| n \tau}+\mathrm{e}^{|c|(n-1) \tau}\right)+(|c| \tau)^{2}\left(\mathrm{e}^{\alpha|c| n \tau}+\mathrm{e}^{|c|(n-1) \tau}\right) \\
& +|c|^{2} \int_{n \tau}^{(n+1) \tau} \int_{s-\tau}^{s} \mathrm{e}^{c \sigma} \mathrm{d} \sigma \mathrm{d} s \\
& \stackrel{\text { (IV) }}{\leq}|c| \tau\left(\mathrm{e}^{\alpha|c| n \tau}+\mathrm{e}^{|c|(n-1) \tau}\right)+(|c| \tau)^{2}\left(\mathrm{e}^{\alpha|c| n \tau}+\mathrm{e}^{|c|(n-1) \tau}\right) \\
& +|c|^{2} \int_{n \tau}^{(n+1) \tau} \int_{s-\tau}^{s} \mathrm{e}^{\alpha|c| n \tau+|c| \tau} \mathrm{d} \sigma \mathrm{d} s \\
& =|c| \tau\left(\mathrm{e}^{\alpha|c| n \tau}+\mathrm{e}^{|c|(n-1) \tau}\right)+(|c| \tau)^{2}\left(\mathrm{e}^{\alpha|c| n \tau}+\mathrm{e}^{|c|(n-1) \tau}\right)+|c|^{2} \tau^{2} \mathrm{e}^{\alpha|c| n \tau+|c| \tau} \\
& =|c| \tau\left(\mathrm{e}^{\alpha|c| n \tau}\left(1+|c| \tau+|c| \tau \mathrm{e}^{|c| \tau}\right)+\mathrm{e}^{|c|(n-1) \tau}(1+|c| \tau)\right) \\
& \stackrel{(\mathrm{V})}{\leq}|c| \tau\left(\mathrm{e}^{\alpha|c|(n+1) \tau}+\mathrm{e}^{|c| n \tau}\right) \text {. }
\end{aligned}
$$

$170 \quad$ Now, we justify Steps (II)-(V) previously applied.

171 Step (II): By the FCT

$$
\int_{n \tau}^{t} \frac{\mathrm{d}}{\mathrm{d} s}\left(\mathrm{e}_{\tau}^{c, s}-\mathrm{e}^{c s}\right) \mathrm{d} s=\mathrm{e}_{\tau}^{c, t}-\mathrm{e}^{c t}-\mathrm{e}_{\tau}^{c, n \tau}+\mathrm{e}^{c n \tau}
$$


Then by the induction hypothesis and taking into account that $t \leq(n+1) \tau$, we have

$$
\begin{aligned}
\left|\mathrm{e}_{\tau}^{c, t}-\mathrm{e}^{c t}\right| & \leq\left|\mathrm{e}_{\tau}^{c, n \tau}-\mathrm{e}^{c n \tau}\right|+\int_{n \tau}^{t}\left|\frac{\mathrm{d}}{\mathrm{d} s}\left(\mathrm{e}_{\tau}^{c, s}-\mathrm{e}^{c s}\right)\right| \mathrm{d} s \\
& \leq|c| \tau\left(\mathrm{e}^{\alpha|c| n \tau}+\mathrm{e}^{|c|(n-1) \tau}\right)+\int_{n \tau}^{(n+1) \tau}\left|\frac{\mathrm{d}}{\mathrm{d} s}\left(\mathrm{e}_{\tau}^{c, s}-\mathrm{e}^{c s}\right)\right| \mathrm{d} s .
\end{aligned}
$$

Step (III): In this step we apply both the FCT and the hypothesis of induction (14).

Step (IV): Following the same argument of Step (I), being $\alpha>1$

$$
c \sigma \leq|c| \sigma \leq|c| s \leq|c|(n+1) \tau \leq \alpha|c| n \tau+|c| \tau,
$$

So, $\mathrm{e}^{c \sigma} \leq \mathrm{e}^{\alpha|c| n \tau+|c| \tau}$.

Step (V): Applying that $\mathrm{e}^{p} \geq 1+p, \forall p \geq 0$ and taking $\alpha=1+\mathrm{e}^{|c| \tau_{0}}$, as $0<\tau \leq \tau_{0}$, then

$$
1+|c| \tau+|c| \tau \mathrm{e}^{|c| \tau}=1+|c| \tau\left(1+\mathrm{e}^{|c| \tau}\right) \leq 1+|c| \tau\left(1+\mathrm{e}^{|c| \tau_{0}}\right)=1+\alpha|c| \tau \leq \mathrm{e}^{\alpha|c| \tau}
$$

And analogously, $1+|c| \tau \leq \mathrm{e}^{|c| \tau}$.

This concludes the proof.

Before showing the convergence (9), we need to establish some technical results that will be required in the subsequent analysis.

Let $c \in \mathbb{R}$ be arbitrary and let us take $\tau_{0} \rightarrow 0^{+}$(thus $\tau \rightarrow 0^{+}$, too) in Theorem 3 . Then

$$
\lim _{\tau \rightarrow 0^{+}} \mathrm{e}_{\tau}^{c, t}=\mathrm{e}^{c t}, \quad t \in[0, T],
$$

where, according to Definition 3,t $=(n-1) \tau, n \rightarrow+\infty$ and $\tau \rightarrow 0^{+}$. Furthermore, as the delayed exponential function is continuous, one derives that

$$
\text { if } \lim _{\tau \rightarrow 0^{+}} f(\tau)=\hat{f} \text {, then } \lim _{\tau \rightarrow 0^{+}} \mathrm{e}_{\tau}^{f(\tau), t}=\mathrm{e}^{\hat{f} t} \neq 0 .
$$

In particular, if we take $f(\tau)=\mathrm{e}^{-a \tau} b$ with $a, b \in \mathbb{R}$ fixed, as $\mathrm{e}^{-a \tau} b \stackrel{\tau \rightarrow 0^{+}}{\longrightarrow} b$ in (16), then

$$
\lim _{\tau \rightarrow 0^{+}} \mathrm{e}_{\tau}^{-a \tau} b, t=\mathrm{e}^{b t} \neq 0
$$

Therefore, there exists $\tau_{1}>0$ such that

$$
\mathrm{e}_{\tau}^{b_{1}, t} \neq 0, b_{1}=\mathrm{e}^{-a \tau} b, \quad \forall \tau \in\left[0, \tau_{1}\right] .
$$

As a consequence of the continuity of the logarithm function and of the delayed exponential function (15), one further obtains

$$
\lim _{\tau \rightarrow 0^{+}} \ln \left(\frac{x \mathrm{e}^{-a t}}{\mathrm{e}_{\tau}^{b_{1}, t}}\right)=\ln \left(x \mathrm{e}^{-(a+b) t}\right) .
$$


189

Therefore, for all $\epsilon>0$ there exists $\tau_{2}: 0<\tau_{2} \leq \tau_{1}$ such that

$$
\left.\left|\ln \left(\frac{x \mathrm{e}^{-a t}}{\mathrm{e}_{\tau}^{b_{1}, t}}\right)-\ln \left(x \mathrm{e}^{-(a+b) t}\right)\right|<\epsilon, \quad \forall \tau \in\right] 0, \tau_{2}[.
$$

To prove the convergence $f(x, t ; \tau) \stackrel{\tau \rightarrow 0^{+}}{\longrightarrow} f(x, t)$ introduced in (9), hereinafter the following hypotheses will be assumed

H1 : The random vector of coefficients $(a(\omega), b(\omega))$ is independent of the $\mathrm{RV} c(\omega)$, i.e.,

$$
f_{c, a, b}(c, a, b)=f_{c}(c) f_{a, b}(a, b) \text {. }
$$

$$
\begin{gathered}
f_{c}(c) \text { is Lipschitz continuous in } \mathbb{R} \text {, i.e., } \\
\exists L_{f_{c}} \geq 0:\left|f_{c}\left(c_{0,1}\right)-f_{c}\left(c_{0,2}\right)\right| \leq L_{f_{c}}\left|c_{0,1}-c_{0,2}\right|, \quad \forall c_{0,1}, c_{0,2} \in \mathbb{R},
\end{gathered}
$$

Let $\tau \in] 0, \tau^{*}\left[: 0<\tau^{*}<\tau_{2}\right.$ and $(x, t) \in \mathcal{D}\left(x_{\tau}(t ; \omega)\right) \cap \mathcal{D}(x(t ; \omega)) \times[(n-1) \tau, n \tau[\subset \mathbb{R} \times[0, T]$ being all of them fixed. Then, taking into account expressions (12) and (13), for $\epsilon^{*}>0$ arbitrary

$$
\begin{aligned}
|f(x, t ; \tau)-f(x, t)| & =\frac{1}{|x|}\left|\int_{\mathbb{R}^{2}}\left[f_{c, a, b}\left(\ln \left(\frac{x \mathrm{e}^{-a t}}{\mathrm{e}_{\tau}^{b_{1}, t}}\right), a, b\right)-f_{c, a, b}\left(\ln \left(x \mathrm{e}^{-(a+b) t}\right), a, b\right)\right] \mathrm{d} a \mathrm{~d} b\right| \\
& \stackrel{\text { H1 }}{=} \frac{1}{|x|}\left|\int_{\mathbb{R}^{2}}\left[f_{c}\left(\ln \left(\frac{x \mathrm{e}^{-a t}}{\mathrm{e}_{\tau}^{b_{1}, t}}\right)\right)-f_{c}\left(\ln \left(x \mathrm{e}^{-(a+b) t}\right)\right)\right] f_{a, b}(a, b) \mathrm{d} a \mathrm{~d} b\right| \\
& \leq \frac{1}{|x|} \int_{\mathbb{R}^{2}}\left|f_{c}\left(\ln \left(\frac{x \mathrm{e}^{-a t}}{\mathrm{e}_{\tau}^{b_{1}, t}}\right)\right)-f_{c}\left(\ln \left(x \mathrm{e}^{-(a+b) t}\right)\right)\right| f_{a, b}(a, b) \mathrm{d} a \mathrm{~d} b \\
& \stackrel{\text { H2 }}{\leq} \frac{L_{f_{c}}}{|x|} \int_{\mathbb{R}^{2}}\left|\ln \left(\frac{x \mathrm{e}^{-a t}}{\mathrm{e}_{\tau}^{b_{1}, t}}\right)-\ln \left(x \mathrm{e}^{-(a+b) t}\right)\right| f_{a, b}(a, b) \mathrm{d} a \mathrm{~d} b \\
& \stackrel{\text { VI) }}{<} \frac{L_{f_{c}}}{|x|} \epsilon^{*} \frac{|x|}{L_{f_{c}}} \int_{\mathbb{R}^{2}} f_{a, b}(a, b) \mathrm{d} a \mathrm{~d} b=\epsilon^{*},
\end{aligned}
$$

where $b_{1}=\mathrm{e}^{-a \tau} b$. In Step (VI), we have applied (17) with $\epsilon=\epsilon^{*} \frac{|x|}{L_{f_{C}}}>0$, while in the last step we have used that $\int_{\mathbb{R}^{2}} f_{a, b}(a, b) \mathrm{d} a \mathrm{~d} b=1$, since $f_{a, b}(a, b)$ is a PDF.

Summarizing, the following result has been established

Theorem 4. Consider the random discrete delay differential equation (4) with $g(t ; \omega)=\mathrm{e}^{a(\omega) t+c(\omega)}$ and whose solution $S P, x_{\tau}(t ; \omega)$, is given by $(11)$. Assume that $a(\omega), b(\omega)$ and $c(\omega)$ are absolutely continuous RVs being $f_{c, a, b}(c, a, b)$ their joint PDF. Then, the 1-PDF, $f(x, t ; \tau)$, of the solution $S P x_{\tau}(t ; \omega)$ is given by $(12)$ at every time instant $t$ where $x_{\tau}(t ; \omega) \neq 0$. Further assume that hypotheses $\boldsymbol{H} 1$ and $\boldsymbol{H} 2$ hold, then $f(x, t ; \tau)$ converges to the 1-PDF, $f(x, t)$, of the solution SP to the random linear differential equation (8), according to (9).

\section{Case II: Computing the PDF of the solution SP and study of the convergence}

In this section we will analyze Case II following an analogous structure to the one exhibited in Section 2. Thus, we will consider random IVP (4) assuming that the initial condition is given 
by a random polynomial of degree $m$

$$
g(t, \omega)=\sum_{j=0}^{m} c_{j}(\omega) t^{j}, \quad m \geq 0
$$

217 and

where $c_{j}(\omega), j=0,1, \ldots, m$ are absolutely continuous RVs defined on a common complete probability space $(\Omega, \mathcal{F}, \mathbb{P})$. For the sake of generality in the subsequent study, we will assume no independence among the involved random inputs, i.e., henceforth, we will assume that $f_{\mathbf{w}}(\mathbf{w})$ is the PDF of the random vector $\mathbf{w}(\omega)=\left(c_{0}(\omega), c_{1}(\omega), \ldots, c_{m}(\omega), a(\omega), b(\omega)\right)$ made of polynomial coefficients, $c_{j}(\omega), 0 \leq j \leq m$ of the initial condition given by (18), and of coefficients $a(\omega)$ and $b(\omega)$ of the random delayed differential equation (4).

\subsection{Computing the PDF}

In this case, according to expression (7), the solution SP $x_{\tau}(t ; \omega)$ is given by

$$
x_{\tau}(t ; \omega)=\varphi_{\tau}^{0}(t, \omega) c_{0}(\omega)+\sum_{j=1}^{m} \varphi_{\tau}^{j}(t, \omega) c_{j}(\omega)
$$

being

$$
\varphi_{\tau}^{0}(t, \omega)=\mathrm{e}^{a(\omega)(t+\tau)} \mathrm{e}_{\tau}^{b_{1}(\omega), t}-\int_{-\tau}^{0} a(\omega) \mathrm{e}^{a(\omega)(t-s)} \mathrm{e}_{\tau}^{b_{1}(\omega), t-\tau-s} \mathrm{~d} s,
$$

$$
\varphi_{\tau}^{j}(t, \omega)=\mathrm{e}^{a(\omega)(t+\tau)} \mathrm{e}_{\tau}^{b_{1}(\omega), t}(-\tau)^{j}+\int_{-\tau}^{0} \mathrm{e}^{a(\omega)(t-s)} \mathrm{e}_{\tau}^{b_{1}(\omega), t-\tau-s}(j-a(\omega) s) s^{j-1} \mathrm{~d} s, \quad j=1,2 \ldots, m,
$$

where $b_{1}(\omega)=\mathrm{e}^{-a(\omega) \tau} b(\omega)$ and $\tau>0$ is a fixed delay.

Remark 1. Notice that, in order to have a non-trivial solution, at least one coefficient $c_{j}(\omega)$, in the initial condition $\sum_{j=0}^{m} c_{j}(\omega) t^{j}$ must be, in the probabilistic sense, different from zero. This fact is guaranteed since $c_{j}(\omega), j=0,1, \ldots, m$, are absolutely continuous RVs.

Let $t \in[(n-1) \tau, n \tau$ [ be fixed, and let us apply the RVT method (Theorem 2) to obtain the PDF of the solution SP, $x_{\tau}(t ; \omega)$, in terms of the PDF, $f_{\mathbf{w}}(\mathbf{w})$, of the random vector $\mathbf{w}(\omega)=$ $\left(c_{0}(\omega), c_{1}(\omega), \ldots, c_{m}(\omega), a(\omega), b(\omega)\right)$. To this end, we will define the following mapping $\mathbf{r}$ : $\mathbb{R}^{m+3} \longrightarrow \mathbb{R}^{m+3}$ whose components are defined by

$$
\begin{aligned}
& x_{1}=r_{1}\left(c_{0}, c_{1}, \ldots, c_{m}, a, b\right)=\varphi_{\tau}^{0}(t, a, b) c_{0}+\sum_{j=1}^{m} \varphi_{\tau}^{j}(t, a, b) c_{j}, \\
& x_{2}=r_{2}\left(c_{0}, c_{1}, \ldots, c_{m}, a, b\right)=c_{1}, \\
& x_{3}=r_{3}\left(c_{0}, c_{1}, \ldots, c_{m}, a, b\right)=c_{2} \text {, } \\
& \vdots \quad \vdots \quad \vdots \\
& x_{m+1}=r_{m+1}\left(c_{0}, c_{1}, \ldots, c_{m}, a, b\right)=c_{m}, \\
& x_{m+2}=r_{m+2}\left(c_{0}, c_{1}, \ldots, c_{m}, a, b\right)=a \text {, } \\
& x_{m+3}=r_{m+3}\left(c_{0}, c_{1}, \ldots, c_{m}, a, b\right)=b \text {. }
\end{aligned}
$$


Observe that, for the sake of clarity, in the previous expression we have emphasized that $\varphi_{\tau}^{j}$ depends on $a$ and $b$. The inverse mapping of $\mathbf{r}$ is $\mathbf{s}: \mathbb{R}^{m+3} \longrightarrow \mathbb{R}^{m+3}$ whose components are

$$
\begin{aligned}
& c_{0}=s_{1}\left(x_{1}, x_{2}, \ldots, x_{m+3}\right)=\frac{x_{1}-\sum_{j=1}^{m} \varphi_{\tau}^{j}\left(t, x_{m+2}, x_{m+3}\right) x_{j+1}}{\varphi_{\tau}^{0}\left(t, x_{m+2}, x_{m+3}\right)}, \\
& c_{1}=s_{1}\left(x_{1}, x_{2}, \ldots, x_{m+3}\right)=x_{2} \text {, } \\
& c_{2}=s_{2}\left(x_{1}, x_{2}, \ldots, x_{m+3}\right)=x_{3} \text {, } \\
& \text { : } \quad \vdots \quad \vdots \\
& c_{m}=s_{m+1}\left(x_{1}, x_{2}, \ldots, x_{m+3}\right)=x_{m+1} \text {, } \\
& a=s_{m+2}\left(x_{1}, x_{2}, \ldots, x_{m+3}\right)=x_{m+2} \text {, } \\
& b=s_{m+3}\left(x_{1}, x_{2}, \ldots, x_{m+3}\right)=x_{m+3} \text {. }
\end{aligned}
$$

The Jacobian of mapping $\mathbf{s}$ is given by

$$
\left|J_{m+3}\right|=\frac{1}{\left|\varphi_{\tau}^{0}\left(t, x_{m+2}, x_{m+3}\right)\right|} \neq 0 .
$$

Notice that, the absolute value of the Jacobian is well-defined since $a(\omega)$ and $b(\omega)$ are absolutely continuous RVs, thus by (20) $\varphi_{\tau}^{0}\left(t, x_{m+2}, x_{m+3}\right)$ is different from zero with probability one. Therefore, the PDF of the random vector $\mathbf{x}(\omega)=\left(x_{1}(\omega), x_{2}(\omega), \ldots, x_{m+3}(\omega)\right)$ in terms of the PDF of $\mathbf{w}(\omega)=\left(c_{0}(\omega), c_{1}(\omega), \ldots, c_{m}(\omega), a(\omega), b(\omega)\right)$ is given by

$$
f_{\mathbf{x}}(\mathbf{x})=f_{\mathbf{w}}\left(\frac{x_{1}-\sum_{j=1}^{m} \varphi_{\tau}^{j}\left(t, x_{m+2}, x_{m+3}\right) x_{j+1}}{\varphi_{\tau}^{0}\left(t, x_{m+2}, x_{m+3}\right)}, x_{2}, \ldots, x_{m+3}\right) \frac{1}{\left|\varphi_{\tau}^{0}\left(t, x_{m+2}, x_{m+3}\right)\right|} .
$$

Then, marginalizing with respect to the random vector $\left(x_{2}(\omega), x_{3}(\omega), \ldots, x_{m+3}(\omega)\right)=\left(c_{1}(\omega)\right.$, $\left.\ldots, c_{m}(\omega), a(\omega), b(\omega)\right)$, given $\tau>0$ and taking $t \in[(n-1) \tau, n \tau[$ arbitrary, the 1-PDF of the solution $\mathrm{SP} x_{\tau}(t ; \omega)$ becomes

$$
f(x, t ; \tau)=\int_{\mathbb{R}^{m+2}} f_{\mathbf{w}}\left(\frac{x-\sum_{j=1}^{m} \varphi_{\tau}^{j}(t, a, b) c_{j}}{\varphi_{\tau}^{0}(t, a, b)}, c_{1}, \ldots, c_{m}, a, b\right) \frac{1}{\left|\varphi_{\tau}^{0}(t, a, b)\right|} \mathrm{d} b \mathrm{~d} a \mathrm{~d} c_{m} \cdots \mathrm{d} c_{1}
$$

\subsection{Convergence}

This subsection is addressed to study conditions in order to the 1-PDF, $f(x, t ; \tau)$, given by (22), that corresponds to random IVP (4) with delay, converges to the 1-PDF, $f(x, t)$, of the solution SP of the corresponding non-delayed random IVP

$$
\left\{\begin{array}{l}
x^{\prime}(t ; \omega)=(a(\omega)+b(\omega)) x(t ; \omega), \quad t \geq 0 \\
x(0 ; \omega)=c_{0}(\omega)
\end{array}\right.
$$

To compute the 1-PDF, $f(x, t)$, notice that the solution SP of random IVP (23) is given by

$$
x(t ; \omega)=c_{0}(\omega) \mathrm{e}^{(a(\omega)+b(\omega)) t} .
$$

Then applying the RVT method (see Theorem 2), it is straightforward to check that

$$
f(x, t)=\int_{\mathbb{R}^{2}} f_{c_{0}, a, b}\left(x \mathrm{e}^{-(a+b) t}, a, b\right) \mathrm{e}^{-(a+b) t} \mathrm{~d} b \mathrm{~d} a .
$$


Here, $f_{c_{0}, a, b}\left(c_{0}, a, b\right)$ stands for the joint PDF of the RVs $c_{0}(\omega), a(\omega)$ and $b(\omega)$, which is obtained by marginalizing the PDF, $f_{c_{0}, c_{1}, \ldots, c_{m}, a, b}\left(c_{0}, c_{1}, \ldots, c_{m}, a, b\right)$, with respect to the RVs $c_{1}(\omega), \ldots, c_{m}(\omega)$ (notice that by hypothesis this PDF is known),

$$
f_{c_{0}, a, b}\left(c_{0}, a, b\right)=\int_{\mathbb{R}^{m}} f_{c_{0}, c_{1}, \ldots, c_{m}, a, b}\left(c_{0}, c_{1}, \ldots, c_{m}, a, b\right) \mathrm{d} c_{m} \cdots \mathrm{d} c_{1} .
$$

To prove the convergence $f(x, t ; \tau) \stackrel{\tau \rightarrow 0^{+}}{\longrightarrow} f(x, t)$ introduced in (9), hereinafter the following hypotheses will be assumed

$$
\text { H1 : } \begin{gathered}
c_{0}(\omega), c_{1}(\omega), \ldots, c_{m}(\omega), a(\omega), b(\omega) \text { are independent RVs, i.e., } \\
f_{c_{0}, c_{1}, \ldots, c_{m}, a, b}\left(c_{0}, c_{1}, \ldots, c_{m}, a, b\right)=f_{c_{0}}\left(c_{0}\right) f_{c_{1}}\left(c_{1}\right) \cdots f_{c_{m}}\left(c_{m}\right) f_{a}(a) f_{b}(b) .
\end{gathered}
$$

H2

$f_{c_{0}}\left(c_{0}\right)$ is Lipschitz continuous in $\mathbb{R}$, i.e.,

$$
\exists L_{f_{c_{0}}}:\left|f_{c_{0}}\left(c_{0,1}\right)-f_{c_{0}}\left(c_{0,2}\right)\right| \leq L_{f_{c_{0}}}\left|c_{0,1}-c_{0,2}\right|, \quad \forall c_{0,1}, c_{0,2} \in \mathbb{R} .
$$

Let $\tau \in] 0, \tau^{*}\left[\right.$, where $\tau^{*}$ will be specified later and $(x, t) \in \mathcal{D}\left(x_{\tau}(t ; \omega)\right) \cap \mathcal{D}(x(t ; \omega)) \times[(n-$ 1) $\tau, n \tau[\subset \mathbb{R} \times[0, T]$ fixed, then taking into account (22) and (24)

$$
\begin{aligned}
& |f(x, t ; \tau)-f(x, t)|=\mid \int_{\mathbb{R}^{m+2}} f_{c_{0}, c_{1}, \ldots, c_{m}, a, b}\left(\frac{x-\sum_{j=1}^{m} \varphi_{\tau}^{j}(t, a, b) c_{j}}{\varphi_{\tau}^{0}(t, a, b)}, c_{1}, \ldots, c_{m}, a, b\right) \frac{1}{\left|\varphi_{\tau}^{0}(t, a, b)\right|} \mathrm{d} b \mathrm{~d} a \mathrm{dc} c_{\mathrm{m}} \cdots \mathrm{d} c_{1} \\
& -\int_{\mathbb{R}^{2}} f_{c_{0}, a, b}\left(x \mathrm{e}^{-(a+b) t}, a, b\right) \mathrm{e}^{-(a+b) t} \mathrm{~d} b \mathrm{~d} a \\
& \stackrel{\text { VII })}{\leq} \int_{\mathbb{R}^{m+2}} \mid f_{c_{0}, c_{1}, \ldots, c_{m}, a, b}\left(\frac{x-\sum_{j=1}^{m} \varphi_{\tau}^{j}(t, a, b) c_{j}}{\varphi_{\tau}^{0}(t, a, b)}, c_{1}, \ldots c_{m}, a, b\right) \frac{1}{\left|\varphi_{\tau}^{0}(t, a, b)\right|} \\
& -f_{c_{0}, c_{1}, \ldots, c_{m}, a, b}\left(x \mathrm{e}^{-(a+b) t}, c_{1}, \ldots, c_{m}, a, b\right) \mathrm{e}^{-(a+b) t} \mid \mathrm{d} b \mathrm{~d} a \mathrm{~d} c_{m} \cdots \mathrm{d} c_{1} \\
& \stackrel{\hat{\mathbf{H}} \mathbf{1}}{=} \int_{\mathbb{R}^{m+2}} \mid f_{c_{0}}\left(\frac{x-\sum_{j=1}^{m} \varphi_{\tau}^{j}(t, a, b) c_{j}}{\varphi_{\tau}^{0}(t, a, b)}\right) \frac{1}{\left|\varphi_{\tau}^{0}(t, a, b)\right|} \\
& -f_{c_{0}}\left(x \mathrm{e}^{-(a+b) t}\right) \mathrm{e}^{-(a+b) t} \mid f_{c_{1}}\left(c_{1}\right) \cdots f_{c_{m}}\left(c_{m}\right) f_{a}(a) f_{b}(b) \mathrm{d} b \mathrm{~d} a \mathrm{~d} c_{m} \cdots \mathrm{d} c_{1} \\
& \leq \int_{\mathbb{R}^{m+2}}\left(\left|f_{c_{0}}\left(\frac{x-\sum_{j=1}^{m} \varphi_{\tau}^{j}(t, a, b) c_{j}}{\varphi_{\tau}^{0}(t, a, b)}\right) \frac{1}{\left|\varphi_{\tau}^{0}(t, a, b)\right|}-f_{c_{0}}\left(\frac{x-\sum_{j=1}^{m} \varphi_{\tau}^{j}(t, a, b) c_{j}}{\varphi_{\tau}^{0}(t, a, b)}\right) \mathrm{e}^{-(a+b) t}\right|\right. \\
& \left.+\left|f_{c_{0}}\left(\frac{x-\sum_{j=1}^{m} \varphi_{\tau}^{j}(t, a, b) c_{j}}{\varphi_{\tau}^{0}(t, a, b)}\right) \mathrm{e}^{-(a+b) t}-f_{c_{0}}\left(x \mathrm{e}^{-(a+b) t}\right) \mathrm{e}^{-(a+b) t}\right|\right) \\
& \times f_{c_{1}}\left(c_{1}\right) \cdots f_{c_{m}}\left(c_{m}\right) f_{a}(a) f_{b}(b) \mathrm{d} b \mathrm{~d} a \mathrm{~d} c_{m} \cdots \mathrm{d} c_{1}
\end{aligned}
$$




$$
\begin{gathered}
=\int_{\mathbb{R}^{m+2}}(\underbrace{f_{c_{0}}\left(\frac{x-\sum_{j=1}^{m} \varphi_{\tau}^{j}(t, a, b) c_{j}}{\varphi_{\tau}^{0}(t, a, b)}\right)}_{(\mathrm{A})} \underbrace{\left|\frac{1}{\left|\varphi_{\tau}^{0}(t, a, b)\right|}-\mathrm{e}^{-(a+b) t}\right|}_{(\mathrm{B})} \\
+\underbrace{\left|f_{c_{0}}\left(\frac{x-\sum_{j=1}^{m} \varphi_{\tau}^{j}(t, a, b) c_{j}}{\varphi_{\tau}^{0}(t, a, b)}\right)-f_{c_{0}}\left(x \mathrm{e}^{-(a+b) t}\right)\right|}_{(\mathrm{C})} \mid \underbrace{\mathrm{e}^{-(a+b) t}}_{(\mathrm{D})})
\end{gathered}
$$

$\times f_{c_{1}}\left(c_{1}\right) \cdots f_{c_{m}}\left(c_{m}\right) f_{a}(a) f_{b}(b) \mathrm{d} b \mathrm{~d} a \mathrm{~d} c_{m} \cdots \mathrm{d} c_{1}$

$$
\begin{aligned}
\stackrel{\text { (VIII) }}{<} & \int_{\mathbb{R}^{m+2}}\left[\left(L_{f_{c_{0}}}\left(\epsilon_{0}+\mathrm{e}^{|a| T} \mathrm{e}^{|b| T}\right)\left(|x|+\sum_{j=1}^{m} \epsilon_{j}\left|c_{j}\right|\right)+F_{0}\right) \epsilon_{0}\right. \\
& \left.+L_{f_{c_{0}}}\left(|x| \epsilon_{0}+\left(\epsilon_{0}+\mathrm{e}^{|a| T} \mathrm{e}^{|b| T}\right) \sum_{j=1}^{m} \epsilon_{j}\left|c_{j}\right|\right) \mathrm{e}^{|a| T} \mathrm{e}^{|b| T}\right)
\end{aligned}
$$

$\times f_{c_{1}}\left(c_{1}\right) \cdots f_{c_{m}}\left(c_{m}\right) f_{a}(a) f_{b}(b) \mathrm{d} b \mathrm{~d} a \mathrm{~d} c_{1} \cdots \mathrm{d} c_{m}$

$$
\begin{aligned}
= & \epsilon_{0} \mathbb{E}\left[L_{f_{c_{0}}}\left(\epsilon_{0}+\mathrm{e}^{|a(\omega)| T} \mathrm{e}^{|b(\omega)| T}\right)\left(|x|+\sum_{j=1}^{m} \epsilon_{j}\left|c_{j}(\omega)\right|\right)+F_{0}\right] \\
& +L_{f_{c_{0}}} \mathbb{E}\left[\left(|x| \epsilon_{0}+\left(\epsilon_{0}+\mathrm{e}^{|a(\omega)| T} \mathrm{e}^{|b(\omega)| T}\right) \sum_{j=1}^{m} \epsilon_{j}\left|c_{j}(\omega)\right|\right) \mathrm{e}^{|a(\omega)| T} \mathrm{e}^{|b(\omega)| T}\right]
\end{aligned}
$$$$
=\epsilon_{0} \mathbb{E}\left[\left(L_{f_{c_{0}}}\left(\epsilon_{0}+\mathrm{e}^{|a(\omega)| T} \mathrm{e}^{|b(\omega)| T}\right)\left(|x|+\sum_{j=1}^{m} \epsilon_{j}\left|c_{j}(\omega)\right|\right)+F_{0}\right)\right]
$$$$
+L_{f_{c_{0}}} \mathbb{E}\left[\left(|x| \epsilon_{0} \mathrm{e}^{|a(\omega)| T} \mathrm{e}^{|b(\omega)| T}+\left(\epsilon_{0} \mathrm{e}^{|a(\omega)| T} \mathrm{e}^{|b(\omega)| T}+\mathrm{e}^{2|a(\omega)| T} \mathrm{e}^{2|b(\omega)| T}\right) \sum_{j=1}^{m} \epsilon_{j}\left|c_{j}(\omega)\right|\right)\right]
$$$$
=\epsilon_{0}\left(L_{f_{c_{0}}}\left(\epsilon_{0}+\mathbb{E}\left[\mathrm{e}^{|a(\omega)| T}\right] \mathbb{E}\left[\mathrm{e}^{|b(\omega)| T}\right]\right)\left(|x|+\sum_{j=1}^{m} \epsilon_{j} \mathbb{E}\left[\left|c_{j}(\omega)\right|\right]\right)+F_{0}\right)
$$$$
+L_{f_{c_{0}}}\left(|x| \epsilon_{0} \mathbb{E}\left[\mathrm{e}^{|a(\omega)| T}\right] \mathbb{E}\left[\mathrm{e}^{|b(\omega)| T}\right]+\left(\epsilon_{0} \mathbb{E}\left[\mathrm{e}^{|a(\omega)| T}\right] \mathbb{E}\left[\mathrm{e}^{|b(\omega)| T}\right]\right.\right.
$$$$
\left.\left.+\mathbb{E}\left[\mathrm{e}^{2|a(\omega)| T}\right] \mathbb{E}\left[\mathrm{e}^{2|b(\omega)| T}\right]\right) \sum_{j=1}^{m} \epsilon_{j} \mathbb{E}\left[\left|c_{j}(\omega)\right|\right]\right) .
$$ 


$$
\begin{aligned}
\mid & \int_{\mathbb{R}^{m+2}} f_{c_{0}, c_{1}, \ldots, c_{m}, a, b}\left(\frac{x-\sum_{j=1}^{m} \varphi_{\tau}^{j}(t, a, b) c_{j}}{\varphi_{\tau}^{0}(t, a, b)}\right) \frac{1}{\left|\varphi_{\tau}^{0}(t, a, b)\right|} \mathrm{d} b \mathrm{~d} a \mathrm{~d} c_{m} \cdots \mathrm{d} c_{1} \\
& -\int_{\mathbb{R}^{2}} f_{c_{0}, a, b}\left(x \mathrm{e}^{-(a+b) t}, a, b\right) \mathrm{e}^{-(a+b) t} \mathrm{~d} b \mathrm{~d} a \mid \\
= & \mid \int_{\mathbb{R}^{m+2}} f_{c_{0}, c_{1}, \ldots, c_{m}, a, b}\left(\frac{x-\sum_{j=1}^{m} \varphi_{\tau}^{j}(t, a, b) c_{j}}{\varphi_{\tau}^{0}(t, a, b)}\right) \frac{1}{\left|\varphi_{\tau}^{0}(t, a, b)\right|} \mathrm{d} b \mathrm{~d} a \mathrm{~d} c_{m} \cdots \mathrm{d} c_{1} \\
= & -\int_{\mathbb{R}^{2}}\left(\int_{\mathbb{R}^{m}} f_{c_{0}, c_{1}, \ldots, c_{m}, a, b}\left(x \mathrm{e}^{-(a+b) t}, c_{1}, \ldots, c_{m}, a, b\right) \mathrm{d} c_{1} \cdots \mathrm{d} c_{m}\right) \mathrm{e}^{-(a+b) t} \mathrm{~d} b \mathrm{~d} a \mid \\
& -\int_{\mathbb{R}_{0}, c_{1}, \ldots, c_{m}, a, b}\left(\frac{x-\sum_{j=1}^{m} \varphi_{\tau}^{j}(t, a, b) c_{j}}{\varphi_{\tau}^{0}(t, a, b)}\right) \frac{1}{\left|\varphi_{\tau}^{0}(t, a, b)\right|} \mathrm{d} b \mathrm{~d} a \mathrm{~d} c_{m} \cdots \mathrm{d} c_{1} \\
= & \mid \int_{\mathbb{R}^{m+2}, c_{1}, \ldots, c_{m}, a, b}\left(f_{c_{0}, c_{1}, \ldots, c_{m}, a, b}\left(\frac{\left.x-\mathrm{e}^{-(a+b) t}, c_{1}, \ldots, c_{m}, a, b\right) \mathrm{e}^{-(a+b) t} \mathrm{~d} b \mathrm{~d} a \mathrm{~d} c_{1} \cdots \mathrm{d} c_{m} \mid}{\varphi_{\tau}^{0}(t, a, b)}\right) \frac{1}{\left|\varphi_{\tau}^{0}(t, a, b)\right|}\right. \\
& \left.-f_{c_{0}, c_{1}, \ldots, c_{m}, a, b}\left(x \mathrm{e}^{-(a+b) t}, c_{1}, \ldots, c_{m}, a, b\right) \mathrm{e}^{-(a+b) t}\right) \mathrm{d} b \mathrm{~d} a \mathrm{~d} c_{m} \cdots \mathrm{d} c_{1} \mid \\
\leq & \int_{\mathbb{R}^{m+2}} \mid f_{c_{0}, c_{1}, \ldots, c_{m}, a, b}\left(\frac{x-\sum_{j=1}^{m} \varphi_{\tau}^{j}(t, a, b) c_{j}}{\varphi_{\tau}^{0}(t, a, b)}\right) \frac{1}{\left|\varphi_{\tau}^{0}(t, a, b)\right|} \\
& -f_{c_{0}, c_{1}, \ldots, c_{m}, a, b}\left(x \mathrm{e}^{-(a+b) t}, c_{1}, \ldots, c_{m}, a, b\right) \mathrm{e}^{-(a+b) t} \mid \mathrm{d} b \mathrm{~d} a \mathrm{~d} c_{m} \cdots \mathrm{d} c_{1}, \\
15 & \left.\mid c_{j}\right)
\end{aligned}
$$


which is just the right-hand side of (VII).

Step (VIII): In this step we will prove that (A) and (D) are bounded, and (B) and (C) tend to zero as $\tau \rightarrow 0^{+}$.

- Let us see that expression (B) tends to zero as $\tau \rightarrow 0^{+}$.

According to (20), for each $\omega \in \Omega$, given $a(\omega)=a$ and $b(\omega)=b$, it is known that $\varphi_{\tau}^{0}(t, a, b)=\mathrm{e}^{a(t+\tau)} \mathrm{e}_{\tau}^{b_{1}, t}-a \int_{-\tau}^{0} \mathrm{e}^{a(t-s)} \mathrm{e}_{\tau}^{b_{1}, t-\tau-s} \mathrm{~d} s$, then by the MVT and the convergence of the delayed exponential function to the exponential one, Theorem 3 , we have

$$
\lim _{\tau \rightarrow 0^{+}} \varphi_{\tau}^{0}(t, a, b)=\mathrm{e}^{(a+b) t} .
$$

By continuity, it is clear that $1 / \varphi_{\tau}^{0}(t, a, b) \stackrel{\tau \rightarrow 0^{+}}{\longrightarrow} 1 / \mathrm{e}^{(a+b) t}$, then for all $\epsilon_{0}>0$ there exists $\tau_{1}$ such that for every $\tau \in] 0, \tau_{1}[\cap] 0, \tau_{0}\left[\right.$ (being $\tau_{0}$ defined in Theorem 3 ),

$$
\left|\frac{1}{\varphi_{\tau}^{0}(t, a, b)}-\frac{1}{\mathrm{e}^{(a+b) t}}\right|<\epsilon_{0} .
$$

- Let us see that expression (A) is bounded.

Let $F_{0}=f_{c_{0}}(0)>0$, then by the Lipschitz condition, hypothesis $\hat{\mathbf{H}} \mathbf{1}$,

$$
\begin{aligned}
f_{c_{0}}\left(\frac{x-\sum_{j=1}^{m} \varphi_{\tau}^{j}(t, a, b) c_{j}}{\varphi_{\tau}^{0}(t, a, b)}\right) & \leq L_{f_{c_{0}}} \frac{1}{\left|\varphi_{0}(t, a, b ; \tau)\right|}\left[|x|+\sum_{j=1}^{m}\left|\varphi_{\tau}^{j}(t, a, b) \| c_{j}\right|\right]+F_{0} \\
& \leq L_{f_{c_{0}}}\left(\epsilon_{0}+\mathrm{e}^{|a| T} \mathrm{e}^{|b| T}\right)\left(|x|+\sum_{j=1}^{m} \epsilon_{j}\left|c_{j}\right|\right)+F_{0},
\end{aligned}
$$

where last inequality is justified by formula (25),

$$
\left|\frac{1}{\varphi_{\tau}^{0}(t, a, b)}\right| \leq\left|\frac{1}{\varphi_{\tau}^{0}(t, a, b)}-\frac{1}{\mathrm{e}^{(a+b) t}}\right|+\frac{1}{\mathrm{e}^{(a+b) t}}<\epsilon_{0}+\mathrm{e}^{-(a+b) t} \leq \epsilon_{0}+\mathrm{e}^{|a| T} \mathrm{e}^{|b| T}, \quad t \in[0, T] .
$$

On the other hand, by (21), $\varphi_{\tau}^{j}(t, a, b)=\mathrm{e}^{a(t+\tau)} \mathrm{e}_{\tau}^{b_{1}, t}(-\tau)^{j}+\int_{-\tau}^{0} \mathrm{e}^{a(t-s)} \mathrm{e}_{\tau}^{b_{1}, t-\tau-s}(j-a s) s^{j-1} \mathrm{~d} s$, $1 \leq j \leq m$, then by the MVT, the convergence of the delayed exponential function to the exponential function and, Theorem 3 , we have

$$
\lim _{\tau \rightarrow 0^{+}} \varphi_{\tau}^{j}(t, a, b)=0, \quad t \in[0, T] .
$$

Thus, for each $j=1, \ldots, m$ and $\epsilon_{j}>0$ arbitrary, there exists $\tau_{j+1}>0$ such that for every $\tau \in] 0, \tau_{j+1}[\cap] 0, \tau_{0}[$

$$
\left|\varphi_{\tau}^{j}(t, a, b)\right|<\epsilon_{j} .
$$

- Let us see that expression (C) tends to zero as $\tau \rightarrow 0^{+}$. 
By the Lipschitz condition, hypothesis $\hat{\mathbf{H}}$, and expressions (25)-(26), one derives

$$
\begin{aligned}
& \left|f_{c_{0}}\left(\frac{x-\sum_{j=1}^{m} \varphi_{\tau}^{j}(t, a, b) c_{j}}{\varphi_{\tau}^{0}(t, a, b)}\right)-f_{c_{0}}\left(x \mathrm{e}^{-(a+b) t}\right)\right| \leq L_{f_{c_{0}}}\left|\frac{x-\sum_{j=1}^{m} \varphi_{\tau}^{j}(t, a, b) c_{j}}{\varphi_{\tau}^{0}(t, a, b)}-x \mathrm{e}^{-(a+b) t}\right| \\
& \quad \leq L_{f_{c_{0}}}\left(|x|\left|\frac{1}{\varphi_{\tau}^{0}(t, a, b)}-\mathrm{e}^{-(a+b) t}\right|+\frac{1}{\left|\varphi_{\tau}^{0}(t, a, b ; \tau)\right|} \sum_{j=1}^{m}\left|\varphi_{\tau}^{j}(t, a, b) \| c_{j}\right|\right) \\
& \quad<L_{f_{c_{0}}}\left(|x| \epsilon_{0}+\left(\epsilon_{0}+\mathrm{e}^{|a| T} \mathrm{e}^{|b| T}\right) \sum_{j=1}^{m} \epsilon_{j}\left|c_{j}\right|\right)
\end{aligned}
$$

$277 \quad$ with $\left.\tau \in \cap_{j=0}^{m+1}\right] 0, \tau_{j}[$.

278

- Let us see that expression (D) is bounded. Indeed, it is clear that

$$
\mathrm{e}^{-(a+b) t} \leq \mathrm{e}^{|a| T} \mathrm{e}^{|b| T}, \quad t \in[0, T] .
$$

Then, the right hand-side of the inequality of Step (VIII) is obtained.

Finally, we use the definition of the expectation and the independence between the RVs $a(\omega)$, $b(\omega)$ and $c_{j}(\omega), j=1,2, \ldots, m$, obtaining the last expression.

282

Now, we assume the following hypothesis in order to prove the convergence

$$
\text { ĤH : } \mathbb{E}\left[\left(\mathrm{e}^{|a(\omega)| T}\right)^{2}\right]=k_{a}<+\infty, \quad \mathbb{E}\left[\left(\mathrm{e}^{|b(\omega)| T}\right)^{2}\right]=k_{b}<+\infty, \quad \mathbb{E}\left[\left|c_{j}\right|\right]=k_{j}<+\infty .
$$

Notice that if $\mathbb{E}\left[(y(\omega))^{2}\right]=k_{y}<\infty$, then by the Cauchy-Schwarz inequality $\mathbb{E}[y(\omega)] \leq$ $\mathbb{E}\left[(y(\omega))^{2}\right]^{1 / 2}=k_{y}^{1 / 2}<\infty$.

Therefore, for $\tau \in] 0, \tau^{*}\left[, \tau^{*}=\min \left\{\tau_{j}: 0 \leq j \leq m\right\},(x, t) \in \mathcal{D}\left(x_{\tau}(t ; \omega)\right) \cap \mathcal{D}(x(t ; \omega)) \times[(n-\right.$ $\left.{ }_{286} 1\right) \tau, n \tau[\subset \mathbb{R} \times[0, T]$ all of them being fixed

$$
\begin{aligned}
|f(x, t ; \tau)-f(x, t)| \leq & \epsilon_{0}\left(L_{f_{c_{0}}}\left(\epsilon_{0}+\mathbb{E}\left[\mathrm{e}^{|a(\omega)| T}\right] \mathbb{E}\left[\mathrm{e}^{|b(\omega)| T}\right]\right)\left(|x|+\sum_{j=1}^{m} \epsilon_{j} \mathbb{E}\left[\left|c_{j}(\omega)\right|\right]+F_{0}\right)\right. \\
& +L_{f_{c_{0}}}|x| \epsilon_{0} \mathbb{E}\left[\mathrm{e}^{|a(\omega)| T}\right] \mathbb{E}\left[\mathrm{e}^{|b(\omega)| T}\right] \\
& +L_{f_{c_{0}}}\left(\epsilon_{0} \mathbb{E}\left[\mathrm{e}^{|a(\omega)| T}\right] \mathbb{E}\left[\mathrm{e}^{|b(\omega)| T}\right]+\mathbb{E}\left[\mathrm{e}^{2|a(\omega)| T}\right] \mathbb{E}\left[\mathrm{e}^{2|b(\omega)| T}\right]\right) \sum_{j=1}^{m} \epsilon_{j} \mathbb{E}\left[\left|c_{j}(\omega)\right|\right] \\
\leq & \epsilon_{0}\left(L_{f_{c_{0}}}\left(\epsilon_{0}+k_{a}^{1 / 2} k_{b}^{1 / 2}\right)\left(|x|+\sum_{j=1}^{m} \epsilon_{j} k_{j}\right)+F_{0}\right) \\
& +L_{f_{c_{0}}}\left(|x| \epsilon_{0} k_{a}^{1 / 2} k_{b}^{1 / 2}+\left(\epsilon_{0} k_{a}^{1 / 2} k_{b}^{1 / 2}+k_{a} k_{b}\right) \sum_{j=1}^{m} \epsilon_{j} k_{j}\right) .
\end{aligned}
$$


Summarizing, the following result has been established.

Theorem 5. Consider the random discrete delay differential equation (4) with $g(t ; \omega)$ given by (18) and whose solution $S P, x_{\tau}(t ; \omega)$, is given by (19)-(21). Let us assume that $\mathbf{w}(\omega)=$ $\left(c_{0}(\omega), c_{1}(\omega), \ldots, c_{m}(\omega), a(\omega), b(\omega)\right)$, is an absolutely continuous random vector being $f_{\mathbf{w}}(\mathbf{w})$ their joint PDF. Then, the 1-PDF, $f(x, t ; \tau)$, of the solution $S P x_{\tau}(t ; \omega)$ is given by $(22)$. Further assume that hypotheses $\hat{\mathbf{H}} \mathbf{1}-\hat{\mathbf{H}} \mathbf{3}$ hold, then $f(x, t ; \tau)$ converges to the $1-P D F, f(x, t)$, of the solution SP to the random linear differential equation (23), according to (9).

\section{Numerical examples}

This section is devoted to illustrate our theoretical findings by means of several numerical experiments where the 1-PDF of the solution SP to random IVP (4) is computed in the two cases previously investigated. For the sake the clarity in the presentation, the explicit expressions of the 1-PDFs, in each one of the numerical examples, are reported in Appendix B since their mathematical representation are somewhat cumbersome. It is important to point out that we have chosen a wide variety of probability distributions for the input parameters $a(\omega), b(\omega)$ and $c(\omega)$.

Example 1. Let us consider random IVP (4) whose initial condition has the form $g(t ; \omega)=$ $\mathrm{e}^{a(\omega) t+c(\omega)}$, which corresponds to Case I. We will assume that $a(\omega), b(\omega)$ and $c(\omega)$ are independent continuous absolutely RVs (hence hypothesis $\mathbf{H 1}$ is fulfilled) with the following distributions:

- $a(\omega)$ is a Gaussian $R V$ with zero mean and standard deviation 0.1, i.e., $a(\omega) \sim N(0 ; 0.1)$.

- $b(\omega)$ is a Beta $R V$ with parameters 2 and 3, i.e., $b(\omega) \sim \operatorname{Be}(2 ; 3)$.

- $c(\omega)$ is an Exponential $R V$ with mean 1/20, i.e., $c(\omega) \sim \operatorname{Exp}(20)$.

Since the first derivative of the PDF of $R V c(\omega)$ is bounded, hypothesis $\mathbf{H} 2$ also holds. Therefore, assumptions of Theorem 4 are fulfilled. Now, we check numerically that the 1-PDF, $f(x, t ; \tau)$, of the solution SP of random IVP (4) with $g(t ; \omega)=\mathrm{e}^{a(\omega) t+c(\omega)}$ converges to the 1-PDF, $f(x, t)$, of the solution SP of random IVP (8). To this end, in Figure 1 we have plotted $f(x, t)$ together with $f(x, t ; \tau)$ with different delays $\tau \in\{0.01,0.05,0.1,0.5,2\}$ at different time instants $t=0.1$, $t=0.5$ and $t=1$. From this graphical representation we can observe that $f(x, t ; \tau)$ converges rapidly to $f(x, t)$ as $\tau \rightarrow 0^{+}$. To numerically assess this convergence, in Table 1 we show the error between $f(x, t)$ and $f(x, t ; \tau)$ for the values of the delays and the time instants previously indicated, according to the following error measure

$$
e_{\tau}^{P D F}(t)=\int_{\mathbb{R}}|f(x, t ; \tau)-f(x, t)| \mathrm{d} x .
$$

We observe that for $t$ fixed, the error $e_{\tau}^{P D F}(t)$ decreases as $\tau \rightarrow 0^{+}$, as expected. We also observe that the velocity of the convergence decreases as t increases.

Example 2. In this second example we consider that the initial condition in random IVP (4) is a polynomial of degree $m, g(t ; \omega)=\sum_{j=0}^{m} c_{j}(\omega) t^{j}, m \geq 0$ which corresponds with Case II studied before. We will consider two problems, when the initial condition is a constant $R V$ (i.e., random polynomial of degree $m=0$ ) and when is a random polynomial of degree $m=1$.

Problem 1: $g(t ; \omega)=c_{0}(\omega)$.

Let $T=0.5$ and assume that $a(\omega), b(\omega)$ and $c_{0}(\omega)$ are independent $R V s$ (hence hypothesis $\hat{\mathbf{H} 1}$ is fulfilled) with the following distributions: 

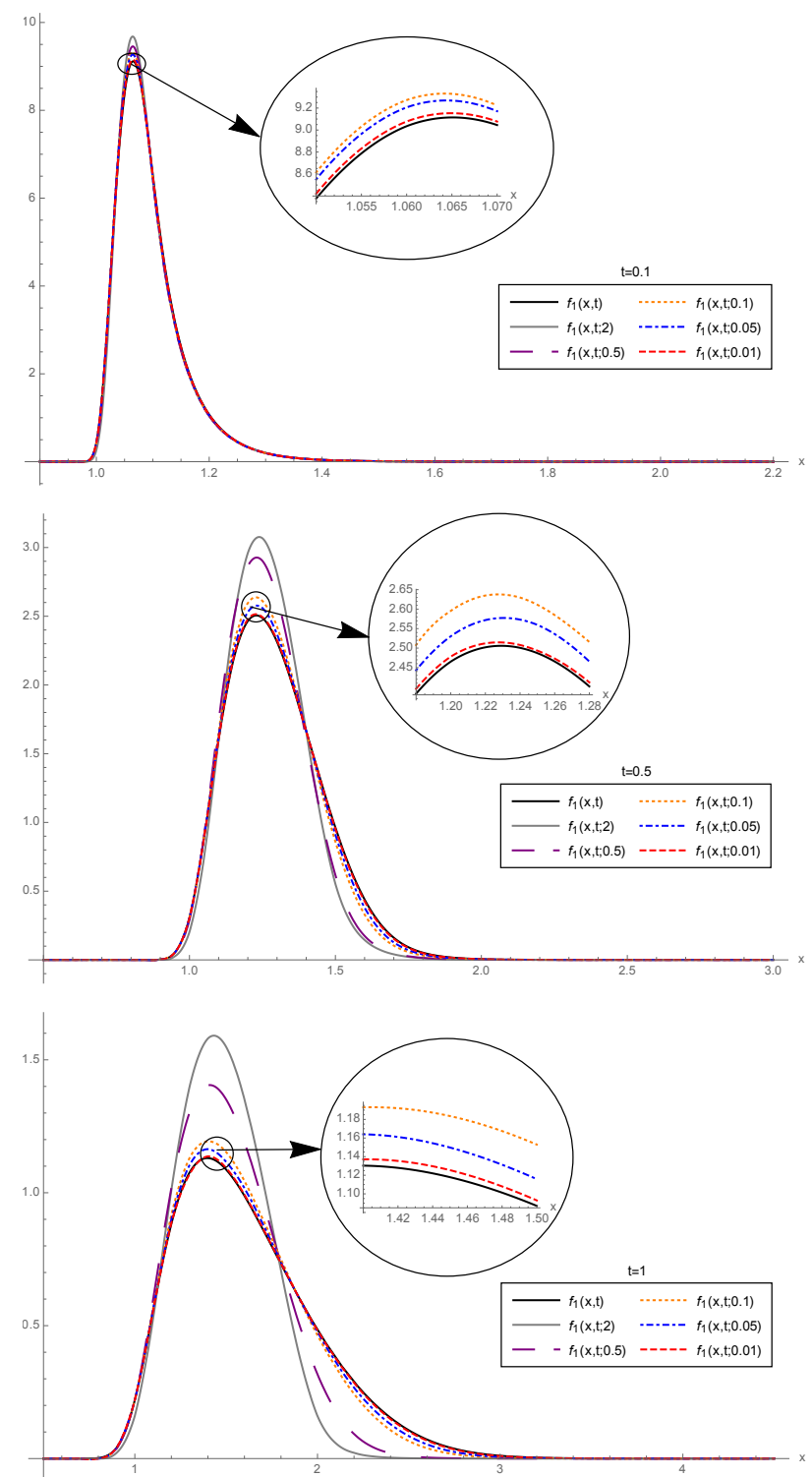

Figure 1: Graphical representation of the PDFs, $f(x, t)$ and $f(x, t ; \tau)$, with different delays $\tau \in\{0.01,0.05,0.1,0.5,2\}$, at different time instants: $t=0.1$ (top), $t=0.5$ (center) and $t=1$ (bottom) in the context of Example 1 . 


\begin{tabular}{|c|c|c|c|c|c|}
\hline$e_{\tau}^{\mathrm{PDF}}(t)$ & $\tau=2$ & $\tau=0.5$ & $\tau=0.1$ & $\tau=0.05$ & $\tau=0.01$ \\
\hline$t=0.10$ & 0.040793 & 0.027599 & 0.021251 & 0.015753 & 0.003949 \\
\hline$t=0.50$ & 0.215938 & 0.172538 & 0.061891 & 0.032553 & 0.006153 \\
\hline$t=1.00$ & 0.382194 & 0.252025 & 0.069224 & 0.035766 & 0.007786 \\
\hline
\end{tabular}

Table 1: Error measure $e_{\tau}^{\mathrm{PDF}}(t)$, defined by (27), with different delays $\tau \in\{0.01,0.05,0.1,0.5,2\}$, at different time instants, $t \in\{0.1,0.5,1\}$, in the context of Example 1 .

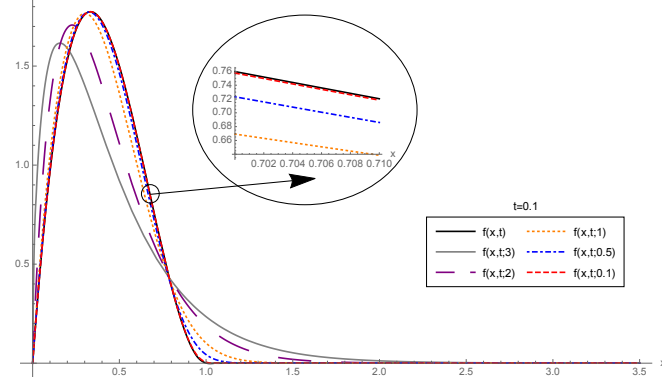
given in hypothesis $\hat{\mathbf{H}} \mathbf{3}$ with $T=0.5$, obtaining
- $a(\omega)$ follows a truncated Gaussian distribution on the interval $I=[-1,1]$ with zero mean and standard deviation 0.1, i.e., $a(\omega) \sim N_{I}(0 ; 0.1)$.

- $b(\omega)$ is an Exponential $R V$ with mean $1 / 50$, i.e., $b(\omega) \sim \operatorname{Exp}(50)$.

- $c_{0}(\omega)$ is a Beta RV with parameters 2 and 3, i.e., $c_{0}(\omega) \sim B e(2 ; 3)$.

Since $f_{c_{0}}^{\prime}\left(c_{0}\right)$ is bounded, hypothesis $\hat{\mathbf{H}} \mathbf{2}$ also holds. Finally, we compute the three values

$$
k_{a}=1.08507, \quad k_{b}=1.02041, \quad k_{0}=0.4,
$$

which are all finite. Therefore, assumptions of Theorem 5 hold. Now, as in the previous example, in order to see the convergence of $f(x, t ; \tau)$ to $f(x, t)$, in Figure 2 we have plotted $f(x, t)$ together with $f(x, t ; \tau)$ with different delays $\tau \in\{0.1,0.5,1,2,3\}$ at the time instants $t=0.1$ and $t=0.5$. In addition, in Table 2 we have calculated the error given in formula (27). From both we can see graphical and numerical the convergence as $\tau \rightarrow 0^{+}$.

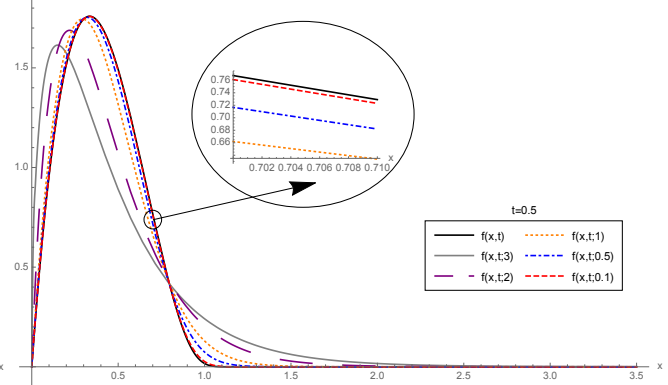

Figure 2: Graphical representation of the PDFs $f(x, t)$ and $f(x, t ; \tau)$, with different delays $\tau \in\{0.1,0.5,1,2,3\}$, at different time instants $t=0.1$ (left) and $t=0.5$ (right) in the context of Problem 1 in Example 2.

In addition, in Figure 3 the mean and the variance of $x(t ; \omega)$ and $x_{\tau}(t ; \omega)$ for different $\tau \in$ $\{0.1,0.5,1,2,3\}$ in the time interval $[0,0.5]$ have been represented. To calculate the mean and the variance of $x_{\tau}(t ; \omega)$ we have used the expressions of $\mathbb{E}\left[x_{\tau}(t ; \omega)\right]$ and $\mathbb{V}\left[x_{\tau}(t ; \omega)\right]$, respectively, given in (6), where $f(x, t ; \tau)$ is defined by (22). In a similar way we have computed the mean and the variance of $x(t ; \omega)$ but using (24). We can observe the convergence as $\tau \rightarrow 0^{+}$. For sake of clarity, the error defined by (28) has been calculated in Table 3. 


\begin{tabular}{|c|c|c|c|c|c|}
\hline$e_{\tau}^{\mathrm{PDF}}(t)$ & $\tau=3$ & $\tau=2$ & $\tau=1$ & $\tau=0.5$ & $\tau=0.1$ \\
\hline$t=0.10$ & 0.425626 & 0.261867 & 0.098525 & 0.028427 & 0.001875 \\
\hline$t=0.50$ & 0.449224 & 0.287860 & 0.115015 & 0.043369 & 0.005402 \\
\hline
\end{tabular}

Table 2: Error measure $e_{\tau}^{\mathrm{PDF}}(t)$, defined by (27), with different delays $\tau \in\{0.1,0.5,1,2,3\}$, at different time instants, $t \in\{0.1,0.5\}$, in the context of Problem 1 of Example 2.

$$
e_{\tau}^{\mathbb{E}}=\int_{0}^{T}\left|\mathbb{E}\left[x_{\tau}(t ; \omega)\right]-\mathbb{E}[x(t ; \omega)]\right| \mathrm{d} t, \quad e_{N}^{\mathbb{V}}=\int_{0}^{T}\left|\mathbb{V}\left[x_{\tau}(t ; \omega)\right]-\mathbb{V}[x(t ; \omega)]\right| \mathrm{d} t .
$$
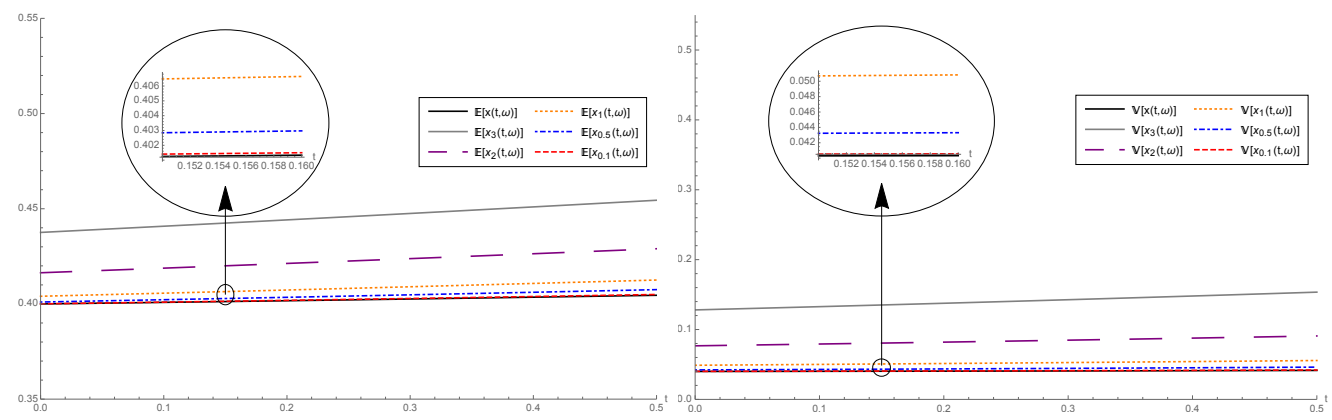

Figure 3: Graphical representation of the mean (left) and the variance (right) of the solutions SP $\left.x_{t} t ; \omega\right)$ and $x_{\tau}(t ; \omega)$, with different delays $\tau \in\{0.01,0.05,0.1,0.5,2\}$, at the time interval $[0,1]$ in the context of Problem 1 in the Example 2.

\begin{tabular}{|c|c|c|c|c|c|}
\hline & $\tau=3$ & $\tau=2$ & $\tau=1$ & $\tau=0.5$ & $\tau=0.1$ \\
\hline$e_{\tau}^{\mathbb{E}}$ & 0.0218545 & 0.0101997 & 0.00301312 & 0.000995992 & 0.00011704 \\
\hline$e_{\tau}^{\bigvee}$ & 0.049849 & 0.0214272 & 0.00574011 & 0.00172316 & 0.000165323 \\
\hline
\end{tabular}

Table 3: Error measures $e_{\tau}^{\mathbb{E}}$ and $e_{\tau}^{\mathbb{V}}$, defined by (28), with different delays $\tau \in\{0.1,0.5,1,2,3\}$, in the context of Problem 1 in Example 2.

Problem 2: $g(t ; \omega)=c_{0}(\omega)+c_{1}(\omega) t$

We take $T=0.5$ and we will assume that $a(\omega), b(\omega), c_{0}(\omega)$ and $c_{1}(\omega)$ are independent $R V s$ (hence hypothesis $\hat{\mathbf{H}} \mathbf{1}$ is fulfilled) with the following distributions:

- $a(\omega)$ is a Beta $R V$ with parameters 2 and 3, i.e., $a(\omega) \sim \operatorname{Be}(2 ; 3)$.

- $b(\omega)$ follows a Gaussian distribution with mean -1 and standard deviation 0.2, i.e., $b(\omega) \sim$ $N(-1 ; 0.2)$.

- $c_{0}(\omega)$ is a Uniform $R V$ in the interval $[-1,1]$, i.e., $c_{0}(\omega) \sim U([-1,1])$. 


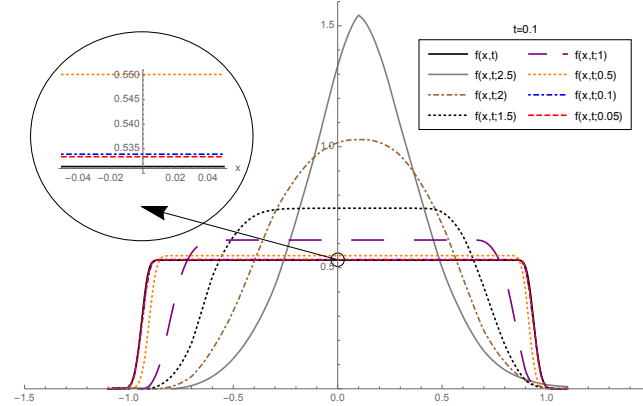
given in hypothesis $\hat{\mathbf{H}} \mathbf{3}$ with $T=0.5$, obtaining

- $c_{1}(\omega)$ follows an Exponential distribution with mean 1/20, i.e., $c_{1}(\omega) \sim \operatorname{Exp}(20)$.

Since $c_{0}(\omega)$ follows a Uniform distribution, then its PDF is a constant and therefore the derivative of the PDF is 0 , then hypothesis $\hat{\mathbf{H}} 2$ also holds. Finally, we compute the three values

$$
k_{a}=1.52247, \quad k_{b}=2.77319, \quad k_{0}=0.5, \quad k_{1}=0.05,
$$

which are all finite. Therefore, assumptions of Theorem 5 are satisfied. Now, as in the previous example, in order to see the convergence of $f(x, t ; \tau)$ to $f(x, t)$, in Figure 4 we have plotted $f(x, t)$ together with $f(x, t ; \tau)$ with different delays $\tau \in\{0.05,0.1,0.5,1,1.5,2,2.5\}$ at time instants $t=$ 0.1 and $t=0.5$. In addition, in Table 4 we have calculated the error given in formula (27). From both we can see graphical and numerical the convergence as $\tau \rightarrow 0^{+}$.

Figure 4: Graphical representation of the PDFs $f(x, t)$ and $f(x, t ; \tau)$, with different delays $\tau \in\{0.05,0.1,0.5,1,1.5,2,2.5\}$ at different time instants $t=0.1$ (left) and $t=0.5$ (right) in the context of Problem 2 in Example 2.

\begin{tabular}{|c|c|c|c|c|c|c|c|}
\hline$e_{\tau}^{\mathrm{PDF}}(t)$ & \multicolumn{2}{|c|}{$\tau=2.5$} & \multicolumn{2}{|c|}{$\tau=2$} & \multicolumn{2}{|c|}{$\tau=1.5$} & $\tau=1$ \\
\hline$t=0.10$ & \multicolumn{2}{|c|}{0.853492} & \multicolumn{2}{|c|}{0.666295} & \multicolumn{2}{|c|}{0.454429} & 0.236062 \\
\hline$t=0.50$ & \multicolumn{2}{|c|}{0.808335} & \multicolumn{2}{|c|}{0.639961} & \multicolumn{2}{|c|}{0.440874} & 0.248945 \\
\hline \multicolumn{2}{|c|}{$e_{\tau}^{\mathrm{PDF}}(t)$} & \multicolumn{2}{|c|}{$\tau=0.5$} & \multicolumn{2}{|c|}{$\tau=0.1$} & \multicolumn{2}{|c|}{$\tau=0.05$} \\
\hline \multicolumn{2}{|c|}{$t=0.10$} & \multicolumn{2}{|c|}{0.0631106} & \multicolumn{2}{|c|}{0.00712427} & \multicolumn{2}{|c|}{0.00581714} \\
\hline \multicolumn{2}{|c|}{$t=0.50$} & \multicolumn{2}{|c|}{0.182441} & \multicolumn{2}{|c|}{0.0520957} & \multicolumn{2}{|c|}{0.0178104} \\
\hline
\end{tabular}

Table 4: Error measure $e_{\tau}^{\mathrm{PDF}}(t)$, defined by (27), with different delays $\tau \in\{0.05,0.1,0.5,1,1.5,2,2.5\}$, at different time instants: $t \in\{0.10,0.50\}$, in the context of Problem 2 in Example 2.

In Figure 5 the mean and the variance of $f(x, t)$ and $f_{1}(x, t ; \tau)$ for different $\tau \in\{0.05,0.1,0.5,1,1.5,2,2.5\}$ in the time interval $[0,0.5]$ have been represented. We can observe the convergence as $\tau \rightarrow 0^{+}$. As in the previous Problem 1, in Table 5 we show the error for the mean and the variance defined by (28). We observe that the difference of these errors becomes smaller as $\tau$ goes to zero. 

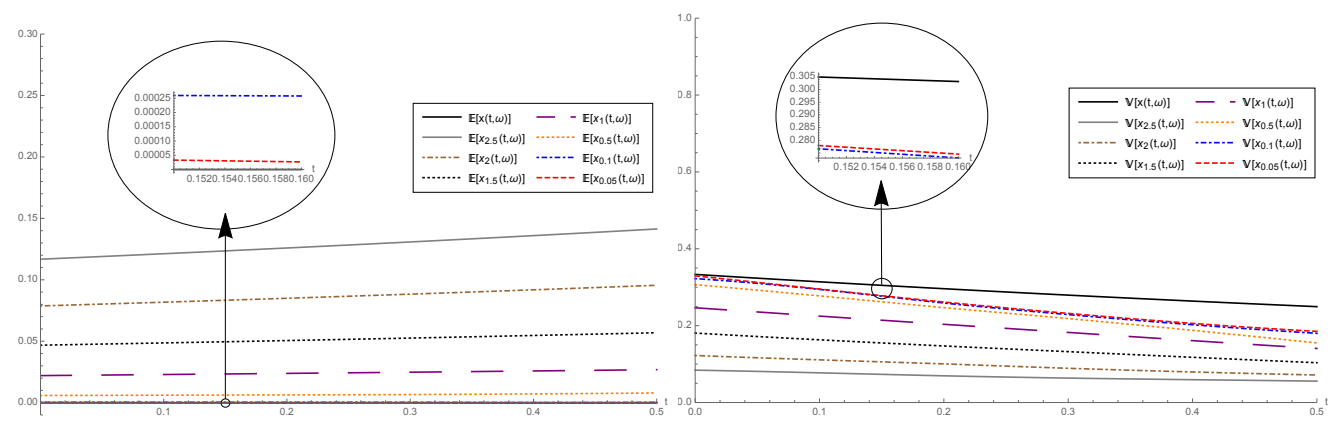

Figure 5: Graphical representation of the mean (left) and the variance (right) of the solutions $\mathrm{SP} x(t ; \omega)$ and $x_{\tau}(t ; \omega)$, with different delays $\tau \in\{0.05,0.1,0.5,1,1.5,2,2.5\}$, at the time interval $[0,0.5]$ in the context of Problem 2 of Example 2 .

\begin{tabular}{|c|c|c|c|c|}
\hline & $\tau=2.5$ & $\tau=2$ & $\tau=1.5$ & $\tau=1$ \\
\hline$e_{\tau}^{\mathbb{E}}$ & 0.0642834 & 0.0434024 & 0.0258283 & 0.0121818 \\
\hline$e_{\tau}^{\mathbb{V}}$ & 0.11049 & 0.0967728 & 0.0742367 & 0.0479234 \\
\hline & & $\tau=0.5$ & $\tau=0.1$ & $\tau=0.05$ \\
\hline & $e_{\tau}^{\mathbb{E}}$ & 0.0033024 & 0.000101439 & 0.000011333 \\
\hline & $e_{\tau}^{\mathbb{V}}$ & 0.0282562 & 0.0208731 & 0.0194024 \\
\hline
\end{tabular}

Table 5: Error measures $e_{\tau}^{\mathbb{E}}$ and $e_{\tau}^{\mathbb{V}}$, defined by (28), with different delays $\tau \in\{0.05,0.1,0.5,1,1.5,2,2.5\}$, in the context of Problem 2 in Example 2. 


\section{Conclusions}

The main goal of the paper has been to compute the first probability density function (1-PDF) of the solution stochastic process of an important class of random linear differential equations with discrete delay. The study has been split into two cases depending on the functional form of the initial condition. It is important to point out that the success of our approach relies upon the knowledge of an explicit expression of the solution. Otherwise, RVT method would need to be combined with numerical strategies. In the examples, we have shown how to compute the mean and the variance of the solution stochastic process from the 1-PDF. It is important to point out that the analysis performed in this contribution is also useful to deal with other types of initial conditions different from the ones presented here. To the best of our knowledge, this is the first time that this class of random differential equations with delay are studied following the proposed approach. In a future contribution, we plan to go a step forward by studying the case which the coefficients of the differential equation are stochastic processes rather than random variables.

\section{Acknowledgements}

This work has been partially supported by the Ministerio de Economía y Competitividad grant MTM2017-89664-P and MTM2015-63723-P and Junta de Andalucía under Proyecto de Excelencia P12-FQM-1492. Ana Navarro Quiles acknowledges the Fundació Ferran Sunyer i Balaguer and the Instituto de Estudios Catalanes for its contribution through the Borsa Ferran Sunyer i Balaguer. Ana Navarro Quiles acknowledges the postdoctoral contract financed by DyCon project funding from the European Research Council (ERC) under the European Unions Horizon 2020 research and innovation programme (grant agreement No 694126-DYCON).

The authors express their deepest thanks and respect to the editors and reviewers for their valuable comments.

\section{Conflict of Interest Statement}

The authors declare that there is no conflict of interests regarding the publication of this article.

\section{Appendix A. Proof of Theorem 1: Existence and uniqueness of the solution}

First, we will show how to construct the solution given in expression (2). To this end, first it is convenient to construct the solution of the following IVP

$$
\left\{\begin{array}{l}
x_{\tau}^{\prime}(t)=b x_{\tau}(t-\tau), \quad t>0, \quad \tau>0 \\
x_{\tau}(t)=1, \quad-\tau \leq t \leq 0
\end{array}\right.
$$

which corresponds to the IVP (1) with $a=0$ and $g(t)=1$. We will see that its solution is the delayed exponential function, $x_{\tau}(t)=\mathrm{e}_{\tau}^{b, t}$. This fact will check in the subintervals $[-\tau, 0],[0, \tau]$, $[\tau, 2 \tau], \ldots$ :

- If $t \in[-\tau, 0]$, it is clear, taken into account the definition of the delayed exponential function (see (3)), that $x_{\tau}(t)=1=\mathrm{e}_{\tau}^{b, t},-\tau \leq t \leq 0$, satisfies the IVP (A.1). 
- If $t \in[0, \tau]$, we integrate the DDE in (A.1) on the interval $[0, t]$

$$
\int_{0}^{t} x_{\tau}^{\prime}(s) \mathrm{d} s=\int_{0}^{t} b x_{\tau}(s-\tau) \mathrm{d} s .
$$

As $s \in[0, t]$ and $t \in[0, \tau], s-\tau \in[-\tau, 0]$, then by the definition of the delayed exponential function $x_{\tau}(s-\tau)=1$. Therefore, applying the FCT

$$
x_{\tau}(t)-x_{\tau}(0)=\int_{0}^{t} b \mathrm{~d} s \stackrel{x_{\tau}(0)=1}{\Longrightarrow} \quad x_{\tau}(t)=1+b t=\mathrm{e}_{\tau}^{b, t}, \quad 0 \leq t \leq \tau .
$$

- If $t \in[\tau, 2 \tau]$, we integrate the DDE in (A.1) on the interval $[\tau, t]$

$$
\int_{\tau}^{t} x_{\tau}^{\prime}(s) \mathrm{d} s=\int_{\tau}^{t} b x_{\tau}(s-\tau) \mathrm{d} s .
$$

Analogously, $s \in[\tau, t]$ and $t \in[\tau, 2 \tau]$ implies $s-\tau \in[0, \tau]$, then, by the previous step, $x_{\tau}(s-\tau)=1+b(s-\tau)$. Therefore, applying the FCT and integrating one gets

$$
x_{\tau}(t)-x_{\tau}(\tau)=\int_{\tau}^{t} b(1+b(s-\tau)) \mathrm{d} s \stackrel{x_{\tau}(\tau)=1+b \tau}{\Longrightarrow} x_{\tau}(t)=1+b t+b^{2} \frac{(t-\tau)^{2}}{2}=\mathrm{e}_{\tau}^{b, t}, \quad \tau \leq t \leq 2 \tau .
$$

Following this reasoning, it is straightforward we conclude by induction that the solution of IVP (A.1) is given by $x_{\tau}(t)=\mathrm{e}_{\tau}^{b, t}$, for each $t \in[(n-1) \tau, n \tau], n=0,1,2, \ldots$.

On the other hand, suppose that the initial condition in (A.1) is a time-dependent function $x_{\tau}(t)=$ $g(t)$, with $-\tau \leq t \leq 0$. By the method of variation of constants [], the solution can be written as

$$
x_{\tau}(t)=x_{\tau}^{*}(t) c+\int_{-\tau}^{0} x_{\tau}^{*}(t-\tau-s) \phi^{\prime}(s) \mathrm{d} s,
$$

being $x_{\tau}^{*}(t)=\mathrm{e}_{\tau}^{b, t}$ the solution of (A.1), $c$ an unknown constant and $\phi(s)$ is an unknown continuously differentiable function which must be determined. If $-\tau \leq t \leq 0$, then $x_{\tau}(t)=g(t)$. Thus, substituting in (A.2)

$$
g(t)=x_{\tau}^{*}(t) c+\int_{-\tau}^{0} x_{\tau}^{*}(t-\tau-s) \phi^{\prime}(s) \mathrm{d} s, \quad-\tau \leq t \leq 0 .
$$

2 We split this integral into the two following integrals

$$
g(t)=x_{\tau}^{*}(t) c+\int_{-\tau}^{t} x_{\tau}^{*}(t-\tau-s) \phi^{\prime}(s) \mathrm{d} s+\int_{t}^{0} x_{\tau}^{*}(t-\tau-s) \phi^{\prime}(s) \mathrm{d} s .
$$

${ }_{3}$ Now, by the properties of the delayed exponential function $x_{\tau}^{*}(t)=\mathrm{e}_{\tau}^{b, t}$ one gets

$$
\text { - }-\tau \leq t \leq 0 \text { implies } x_{\tau}^{*}(t)=1 \text {. }
$$

- When $s \in[-\tau, t]$, then $t-\tau-s \in[-\tau, 0]$. Therefore, $x_{\tau}^{*}(t-\tau-s)=1$.

- When $s \in[t, 0]$, then $t-\tau-s \in[-2 \tau,-\tau]$. Therefore, $x_{\tau}^{*}(t-\tau-s)=0$ if $s \neq t$ and $x_{\tau}^{*}(t-\tau-s)=1$ if $s=t$. 
Thus,

$$
g(t)=c+\int_{-\tau}^{t} \phi^{\prime}(s) \mathrm{d} s \stackrel{\mathrm{FCT}}{=} c+\phi(t)-\phi(-\tau) .
$$

Therefore, we can take in (A.2) $\phi(t)=g(t)$ and $c=g(-\tau)$ so that the previous equation fulfils. Summarizing, the solution is given by

$$
x_{\tau}(t)=\mathrm{e}_{\tau}^{b, t} g(-\tau)+\int_{-\tau}^{0} \mathrm{e}_{\tau}^{b, t-\tau-s} g^{\prime}(s) \mathrm{d} s .
$$

Now, we are ready to build the solution of the IVP (1). To this end, we will follow the same argument as before, so first we will construct the solution of the IVP

$$
\left\{\begin{array}{l}
x_{\tau}^{\prime}(t)=a x_{\tau}(t)+b x_{\tau}(t-\tau), \quad t>0, \quad \tau>0 \\
x_{\tau}(t)=\mathrm{e}^{a t}, \quad-\tau \leq t \leq 0,
\end{array}\right.
$$

where the initial condition $\mathrm{e}^{a t}$ is chosen in order to consider the non-delayed part $a x_{\tau}(t)$. In this case, and by the result obtained for the IVP (A.1), the solution must be of the form $x_{\tau}(t)=$ $\mathrm{e}^{a t} \mathrm{e}_{\tau}^{b_{1}, t}$. We will determine $b_{1}$ by imposing that $x_{\tau}(t)=\mathrm{e}^{a t} \mathrm{e}_{\tau}^{b_{1}, t}$ satisfies the DDE displayed in IVP (A.4):

$$
\begin{aligned}
x_{\tau}^{\prime}(t)=\frac{\mathrm{d}}{\mathrm{d} t}\left(\mathrm{e}^{a t} \mathrm{e}_{\tau}^{b_{1}, t}\right) & =a \mathrm{e}^{a t} \mathrm{e}_{\tau}^{b_{1}, t}+\mathrm{e}^{a t} b_{1} \mathrm{e}_{\tau}^{b_{1}, t-\tau} \\
& =a x_{\tau}(t)+b_{1} \mathrm{e}^{a \tau} \mathrm{e}^{a(t-\tau)} \mathrm{e}_{\tau}^{b_{1}, t-\tau} \\
& =a x_{\tau}(t)+b_{1} \mathrm{e}^{a \tau} x_{\tau}(t-\tau) .
\end{aligned}
$$

Notice that we have used the differentiating rule: $\frac{\mathrm{d}}{\mathrm{d} t}\left(\mathrm{e}_{\tau}^{b_{1}, t}\right)=b_{1} \mathrm{e}_{\tau}^{b_{1}, t-\tau}$. This derivative can be directly checked by computing the derivative of the delayed exponential in each subinterval from its definition (see (3)). Comparing (A.5) with (A.4) is enough taking $b_{1} \mathrm{e}^{a \tau}=b$ so that $x_{\tau}^{*}(t)=\mathrm{e}^{a t} \mathrm{e}_{\tau}^{b_{1}, t}$ with $b_{1}=\mathrm{e}^{-a \tau} b$ be a solution of the DDE of (A.4). As we are interested in computing the solution of the IVP (1) whose initial condition is a time-dependent function $g(t)$, we will use the method of variation of constants again. So, we seek a solution of the IVP (1) in the form,

$$
x_{\tau}(t)=x_{\tau}^{*}(t) c+\int_{-\tau}^{0} x_{\tau}^{*}(t-\tau-s) \phi(s) \mathrm{d} s,
$$

where $x_{\tau}^{*}(t)=\mathrm{e}^{a t} \mathrm{e}_{\tau}^{b_{1}, t}$ with $b_{1}=\mathrm{e}^{-a \tau} b, c$ is an unknown constant and $\phi(s)$ is an unknown continuously differentiable function that must be calculated. As before, we choose $c$ and $\phi(s)$ so that the initial condition is satisfied, $x_{\tau}(t)=g(t),-\tau \leq t \leq 0$, i.e.,

$$
g(t)=x_{\tau}^{*}(t) c+\int_{-\tau}^{0} x_{\tau}^{*}(t-\tau-s) \phi(s) \mathrm{d} s, \quad-\tau \leq t \leq 0 .
$$

To obtain $c$, we set $t=-\tau$. Then, by the definition of the delayed exponential function one gets

- $x_{\tau}^{*}(-\tau)=\mathrm{e}^{-a \tau}$.

- $s \in[-\tau, 0]$, then $t-\tau-s=-2 \tau-s \in[-2 \tau,-\tau]$. Then, $x_{\tau}^{*}(t-\tau-s)=0$ if $-\tau<s \leq 0$ and $x_{\tau}^{*}(t-\tau-s)=1$ if $s=-\tau$.

Therefore, substituting in (A.7), $g(-\tau)=\mathrm{e}^{-a \tau} c$ and isolating $c$ we obtain $c=\mathrm{e}^{a \tau} g(-\tau)$. To determine the function $\phi(t)$, we split the integral in expression (A.7) into two integrals

$$
g(t)=x_{\tau}^{*}(t) c+\int_{-\tau}^{t} x_{\tau}^{*}(t-\tau-s) \phi(s) \mathrm{d} s+\int_{t}^{0} x_{\tau}^{*}(t-\tau-s) \phi(s) \mathrm{d} s .
$$


Following the same reasoning, by the definition of the delayed exponential function one deduces

- $x_{\tau}^{*}(t)=\mathrm{e}^{a t}$.

- In the first integral, $s \in[-\tau, t]$, then $t-\tau-s \in[-\tau, t] \subset[-\tau, 0]$. Then, $x_{\tau}^{*}(t-\tau-s)=\mathrm{e}^{a(t-\tau-s)}$.

- In the second integral, $s \in[t, 0]$, then $t-\tau-s \in[t-\tau,-\tau] \subset[-2 \tau,-\tau]$. Then, $x_{\tau}^{*}(t-\tau-s)=0$ if $t<s \leq 0$ and $x_{\tau}^{*}(t-\tau-s)=\mathrm{e}^{-a \tau}$ if $s=t$.

Therefore, substituting in (A.8) and taking into account that $c=\mathrm{e}^{a \tau} g(-\tau)$,

$$
g(t)=\mathrm{e}^{a(t+\tau)} g(-\tau)+\int_{-\tau}^{t} \mathrm{e}^{a(t-\tau-s)} \phi(s) \mathrm{d} s .
$$

Differentiating relation (A.9), by general Leibniz rule, we obtain

$$
g^{\prime}(t)=a \mathrm{e}^{a(t+\tau)} g(-\tau)+a \int_{-\tau}^{t} \mathrm{e}^{a(t-\tau-s)} \phi(s) \mathrm{d} s+\mathrm{e}^{-a \tau} \phi(t) .
$$

Solving the system (A.9)-(A.10), we obtain

$$
\phi(t)=\mathrm{e}^{a \tau}\left(g^{\prime}(t)-a g(t)\right) .
$$

Finally, substituting $x_{\tau}^{*}(t)=\mathrm{e}^{a t} \mathrm{e}_{\tau}^{b_{1}, t}$ with $b_{1}=\mathrm{e}^{-a \tau} b, c=\mathrm{e}^{a \tau} g(-\tau)$ and $\phi(t)=\mathrm{e}^{a \tau}\left(g^{\prime}(t)-a g(t)\right)$ in (A.6), we obtain the solution given in (2) to the IVP (1).

Uniqueness follows because the function $f(t, x, y)=a x+b y$ defining the right-hand side of the $\operatorname{DDE}(1)$ (with $x \equiv x_{\tau}$ and $y \equiv x_{\tau}(t-\tau)$ ) is continuous with respect to $t$ for every $x$ and $y$, and it is locally Lipschitzian with respect to $x$ and $y$ because is linear [23, p.296].

\section{Appendix B. Explicit expressions for the 1-PDFs in the Examples 1 and 2}

Example 1: $a(\omega), b(\omega)$ and $c(\omega)$ are independent RVs $\left(f_{c, a, b}(c, a, b)=f_{c}(c) f_{a}(a) f_{b}(b)\right)$ with the following distributions

- $a(\omega) \sim N(\mu=0 ; \sigma=0.1)$

$$
f_{a}(a)=\frac{1}{\sqrt{2 \pi \sigma^{2}}} \mathrm{e}^{-\frac{(a-\mu)^{2}}{2 \sigma^{2}}}=\frac{1}{\sqrt{0.02 \pi}} \mathrm{e}^{-\frac{a^{2}}{0.02}}, \quad a \in \mathbb{R} .
$$

${ }_{461} \quad b(\omega) \sim B e(\alpha=2 ; \beta=3)$

$$
\left.f_{b}(b)=\frac{\Gamma(\alpha+\beta)}{\Gamma(\alpha) \Gamma(\beta)} b^{\alpha-1}(1-b)^{\beta-1}=12 b(1-b)^{2}, \quad b \in\right] 0,1[, \quad(0, \text { in otherwise }) .
$$

- $c(\omega) \sim \operatorname{Exp}(\lambda=20)$

$$
f_{c}(c)=\lambda \mathrm{e}^{-\lambda c}=20 \mathrm{e}^{-20 c}, \quad c>0, \quad(0, \text { in otherwise }) .
$$


We compute the 1-PDF of the solution SP of the IVP (8), substituting the above distributions in expression (13)

$$
\begin{aligned}
f(x, t) & =\int_{0}^{1} \int_{-\infty}^{\infty} f_{c}\left(\ln \left(x \mathrm{e}^{-(a+b) t}\right)\right) f_{a}(a) f_{b}(b) \frac{1}{|x|} \mathrm{d} a \mathrm{~d} b \\
& =\int_{0}^{1} \int_{-\infty}^{\infty} f_{c}\left(\ln \left(x \mathrm{e}^{-(a+b) t}\right)\right) \frac{1}{\sqrt{0.02 \pi}} \mathrm{e}^{-\frac{a^{2}}{0.02}} 12 b(1-b)^{2} \frac{1}{|x|} \mathrm{d} a \mathrm{~d} b .
\end{aligned}
$$

Since $c=c(\omega)$ has an Exponential distribution, the last integral must be calculated taking into account the condition $\ln \left(x \mathrm{e}^{-(a+b) t}\right)>0$. This is the reason why we have not performed the formal substitution $f_{c}\left(\ln \left(x \mathrm{e}^{-(a+b) t}\right)\right)=20\left(x \mathrm{e}^{-(a+b) t}\right)^{-20}$ in the previous expression for $f(x, t)$.

Now, we determine the expression of the 1-PDF of the solution SP to the IVP (4), substituting the above distributions in expression (12)

$$
\begin{aligned}
f(x, t ; \tau) & =\int_{0}^{1} \int_{-\infty}^{\infty} f_{c}\left(\ln \left(\frac{x \mathrm{e}^{-a t}}{\mathrm{e}_{\tau}^{b_{1}, t}}\right)\right) f_{a}(a) f_{b}(b) \frac{1}{|x|} \mathrm{d} a \mathrm{~d} b \\
& =\int_{0}^{1} \int_{-\infty}^{\infty} f_{c}\left(\ln \left(\frac{x \mathrm{e}^{-a t}}{\mathrm{e}_{\tau}^{b_{1}, t}}\right)\right) \frac{1}{\sqrt{0.02 \pi}} \mathrm{e}^{-\frac{a^{2}}{0.02}} 12 b(1-b)^{2} \frac{1}{|x|} \mathrm{d} a \mathrm{~d} b,
\end{aligned}
$$

where $b_{1}=\mathrm{e}^{-a \tau} b$. Again, this last double integral must be calculated taking into account the condition $\ln \left(\frac{x \mathrm{e}^{-a t}}{\mathrm{e}_{\tau}, t}\right)>0$.

Example 2, Problem 1: $a(\omega), b(\omega)$ and $c_{0}(\omega)$ are independent RVs with the following distributions

- $a(\omega) \sim N_{I}(\mu=0 ; \sigma=0.1)$, with $I=[\alpha, \beta]=[-1,1]$.

$f_{a}(a)=\frac{\phi\left(\frac{a-\mu}{\sigma}\right)}{\sigma\left(\Phi\left(\frac{\beta-\mu}{\sigma}\right)-\Phi\left(\frac{\alpha-\mu}{\sigma}\right)\right)}, \quad$ where $\phi(x)=\frac{1}{\sqrt{2 \pi}} \mathrm{e}^{-\frac{x^{2}}{2}}, \quad$ and $\quad \Phi(x)=\frac{1}{2}\left(1+\operatorname{erf}\left(\frac{x}{\sqrt{2}}\right)\right)$,

being $\operatorname{erf}(x)$ the error function, defined by $\operatorname{erf}(x)=\frac{2}{\sqrt{\pi}} \int_{0}^{x} \mathrm{e}^{-t^{2}} \mathrm{~d} t$.

Notice that, in this case, it can be proved that $\Phi\left(\frac{\beta-\mu}{\sigma}\right)-\Phi\left(\frac{\alpha-\mu}{\sigma}\right)=1$. Then,

$$
f_{a}(a)=\frac{1}{\sqrt{0.02 \pi}} \mathrm{e}^{-\frac{a^{2}}{0.02}}, \quad a \in[-1,1], \quad(0, \text { in otherwise })
$$

- $b(\omega) \sim \operatorname{Exp}(\lambda=50)$

$$
f_{b}(b)=50 \mathrm{e}^{-50 b}, \quad b>0, \quad(0, \text { in otherwise }) .
$$

- $c_{0}(\omega) \sim B e(\alpha=2 ; \beta=3)$

$$
\left.f_{c_{0}}\left(c_{0}\right)=12 c_{0}\left(1-c_{0}\right)^{2}, \quad c_{0} \in\right] 0,1[, \quad(0, \text { in otherwise }) .
$$


We compute the 1-PDF of the solution SP of the IVP (8), substituting the above distributions in expression (24):

$$
\begin{aligned}
f(x, t) & =\int_{-1}^{1} \int_{0}^{+\infty} f_{c_{0}}\left(x \mathrm{e}^{-(a+b) t}\right) f_{a}(a) f_{b}(b) \mathrm{e}^{-(a+b) t} \mathrm{~d} b \mathrm{~d} a \\
& =\int_{-1}^{1} \int_{0}^{+\infty} f_{c_{0}}\left(x \mathrm{e}^{-(a+b) t}\right) \frac{1}{\sqrt{0.02 \pi}} \mathrm{e}^{-\frac{a^{2}}{0.02}} 50 \mathrm{e}^{-50 b} \mathrm{e}^{-(a+b) t} \mathrm{~d} b \mathrm{~d} a .
\end{aligned}
$$

This integral must be calculated taking into account the restriction $0<x \mathrm{e}^{-(a+b) t}<1$ since $f_{c_{0}}(\cdot)$ is the PDF of a Beta random variable.

Now, we determine the expression of the 1-PDF of the solution SP of the IVP (4), substituting the above distributions in expression (22)

$$
\begin{aligned}
f(x, t ; \tau) & =\int_{-1}^{1} \int_{0}^{+\infty} f_{c_{0}}\left(\frac{x}{\varphi_{\tau}^{0}(t, a, b)}\right) f_{a}(a) f_{b}(b) \frac{1}{\left|\varphi_{\tau}^{0}(t, a, b)\right|} \mathrm{d} b \mathrm{~d} a \\
& =\int_{-1}^{1} \int_{0}^{+\infty} f_{c_{0}}\left(\frac{x}{\varphi_{\tau}^{0}(t, a, b)}\right) \frac{1}{\sqrt{0.02 \pi}} \mathrm{e}^{-\frac{a^{2}}{0.02}} 50 \mathrm{e}^{-50 b} \frac{1}{\left|\varphi_{\tau}^{0}(t, a, b)\right|} \mathrm{d} b \mathrm{~d} a
\end{aligned}
$$

where $\varphi_{\tau}^{0}(t, a, b)$ is defined by (20) being $b_{1}(\omega)=\mathrm{e}^{-a(\omega) \tau} b(\omega), \tau>0$. As $c_{0}(\omega)$ has a Beta distribution, this integral must be calculated taking into account the condition $0<x / \varphi_{\tau}^{0}(t, a, b)<$ 1.

Example 2, Problem 2: $a(\omega), b(\omega), c_{0}(\omega)$ and $c_{1}(\omega)$ are independent RVs with the following distributions

- $a(\omega) \sim B e(\alpha=2 ; \beta=3)$

$$
\left.f_{a}(a)=12 a(1-a)^{2}, \quad a \in\right] 0,1[, \quad(0, \text { in otherwise }) .
$$

- $b(\omega) \sim N(\mu=-1 ; \sigma=0.2)$

$$
f_{b}(b)=\frac{1}{\sqrt{2 \pi \sigma^{2}}} \mathrm{e}^{-\frac{(b a-\mu)^{2}}{2 \sigma^{2}}}=\frac{1}{\sqrt{0.08 \pi}} \mathrm{e}^{-\frac{(b+1)^{2}}{0.08}}, \quad b \in \mathbb{R} .
$$

- $c_{0}(\omega) \sim U\left(\left[u_{1}=-1, u_{2}=1\right]\right)$

$$
\left.f_{c_{0}}\left(c_{0}\right)=\frac{1}{u_{2}-u_{1}}=\frac{1}{2}, \quad c_{0} \in\right]-1,1[, \quad(0, \text { in otherwise }) .
$$

- $c_{1}(\omega) \sim \operatorname{Exp}(\lambda=20)$

$$
f_{c_{1}}\left(c_{1}\right)=20 \mathrm{e}^{-20 c_{1}}, \quad c_{1}>0, \quad(0, \text { in otherwise }) .
$$

We compute the 1-PDF of the solution SP of the IVP (8), substituting the above distributions in expression (24)

$$
\begin{aligned}
f(x, t) & =\int_{0}^{1} \int_{-\infty}^{+\infty} f_{c_{0}}\left(x \mathrm{e}^{-(a+b) t}\right) f_{a}(a) f_{b}(b) \mathrm{e}^{-(a+b) t} \mathrm{~d} b \mathrm{~d} a \\
& =\int_{0}^{1} \int_{-\infty}^{+\infty} f_{c_{0}}\left(x \mathrm{e}^{-(a+b) t}\right) \frac{1}{2 a(1-a)^{2}} \frac{1}{\sqrt{0.08 \pi}} \mathrm{e}^{-\frac{(b+1)^{2}}{0.08}} \mathrm{e}^{-(a+b) t} \mathrm{~d} b \mathrm{~d} a,
\end{aligned}
$$


where this last double integral must be calculated taking into account the condition $-1<x \mathrm{e}^{-(a+b) t}<$ 1 since $c_{0}(\omega) \sim U([-1,1])$.

Now, we determine the expression of the 1-PDF of the solution SP of the IVP (4), substituting the above distributions in expression (22)

$$
\begin{aligned}
f(x, t ; \tau) & =\int_{0}^{+\infty} \int_{0}^{1} \int_{-\infty}^{+\infty} f_{c_{0}}\left(\frac{x-\varphi_{\tau}^{1}(t, a, b) c_{1}}{\varphi_{\tau}^{0}(t, a, b)}\right) f_{c_{1}}\left(c_{1}\right) f_{a}(a) f_{b}(b) \frac{1}{\left|\varphi_{\tau}^{0}(t, a, b)\right|} \mathrm{d} b \mathrm{~d} a \mathrm{~d} c_{1} \\
& =\int_{0}^{+\infty} \int_{0}^{1} \int_{-\infty}^{+\infty} f_{c_{0}}\left(\frac{x-\varphi_{\tau}^{1}(t, a, b) c_{1}}{\varphi_{\tau}^{0}(t, a, b)}\right) 20 \mathrm{e}^{-20 c_{1}} 12 a(1-a)^{2} \frac{1}{\sqrt{0.08 \pi}} \mathrm{e}^{-\frac{(b+1)^{2}}{0.08}} \frac{1}{\left|\varphi_{\tau}^{0}(t, a, b)\right|} \mathrm{d} b \mathrm{~d} a \mathrm{~d} c_{1} \\
& =\int_{0}^{+\infty} \int_{0}^{1} \int_{-\infty}^{+\infty} f_{c_{0}}\left(\frac{x-\varphi_{\tau}^{1}(t, a, b) c_{1}}{\varphi_{\tau}^{0}(t, a, b)}\right) \frac{240}{\sqrt{0.08 \pi}} \mathrm{e}^{-\left(\frac{(b+1)^{2}}{0.08}+20 c_{1}\right)} \frac{a(1-a)^{2}}{\left|\varphi_{\tau}^{0}(t, a, b)\right|} \mathrm{d} b \mathrm{~d} a \mathrm{~d} c_{1},
\end{aligned}
$$

where $\varphi_{\tau}^{0}(t, a, b)$ and $\varphi_{\tau}^{1}(t, a, b)$ are defined by (20) and (21) (for $j=1$ ), being $b_{1}(\omega)=\mathrm{e}^{-a(\omega) \tau} b(\omega)$, $\tau>0$. The last triple integral must be calculated taking into account the following condition $-1<\left(x-\varphi_{\tau}^{1}(t, a, b) c_{1}\right) / \varphi_{\tau}^{0}(t, a, b)<1$ since $c_{0}(\omega) \sim U([-1,1])$.

Remark: Finally, we point out that the integrals defining $f(x, t)$ and $f(x, t ; \tau)$, in Example 1 and in Example 2 (Problems 1 and 2), have been computed numerically by Mathematica ${ }^{\complement}$ package.

\section{References}

[1] D. Y. Khusainov, M. Pokojovy, E. Azizbayov, Classical solvability for linear 1d heat equation with constant delay, Zhurnal Obchyslyuval'nï ta Prykladnoï Matematyky 115 (2014) 76-87.

[2] D. Y. Khusainov, A. F. Ivanov, I. V. Kovarzh, Solution of one heat equation with delay, Nonlinear Ocillations 12 (2009) 260-282. doi:10.1007/s11072-009-0075-3.

[3] T. T. Soong, Random Differential Equations in Science and Engineering, Academic Press, New York, 1973.

[4] S. Liu, A. Debbouche, J. R. Wang, On the iterative learning control for stochastic impulsive differential equations with randomly varying trial lengths, Journal of Computational and Applied Mathematics 312 (2017) 47-57. doi:10.1016/j.cam.2015.10.028.

[5] M. Mourad, A. Debbouche, Complete controllability of nonlocal fractional stochastic differential evolution equations with Poisson jumps in Hilbert spaces, International Journal of Advances in Applied Mathematics in Mechanics 3 (1) (2015) 41-47. doi:10.1016/j.cam.2015.10.028.

[6] L. Shaikhet, Stability of equilibriums of stochastically perturbed delay differential neoclassical growth model, Discrete and Continuous Dynamical Systems. Series B 22 (4) (2015) 1565-1573. doi:10.3934/dcdsb.2017075.

[7] F. J. Santonja, L. Shaikhet, Probabilistic stability analysis of social obesity epidemic by a delayed stochastic model, Nonlinear Analysis: Real World Applications 17 (2014) 114-125. doi:10.1016/j.nonrwa.2013.10.010.

[8] Y. Mishura, T. Shalaiko, G. Shevchenko, Convergence of solutions of mixed stochastic delay differential equations with applications, Applied Mathematics and Computation 257 (2015) 487-497. doi:10.1016/j.amc.2015.01.019.

[9] K. Wu, X. Ding, Convergence and stability of euler method for impulsive stochastic delay differential equations, Applied Mathematics and Computation 229 (2014) 151-158. doi:10.1016/j.amc.2013.12.041.

[10] C. Burgos, J. Calatayud, J. C. Cortés, A. Navarro-Quiles, A full probabilistic solution of the random linear fractional differential equation via the Random Variable Transformation technique, Mathematical Methods in Applied Sciences 41 (2018) 9037-9047. doi:10.1002/mma.4881.

[11] B. Kegan, R. Webster West, Modeling the simple epidemic with deterministic differential equations and random initial conditions, Mathematical Biosciences 195 (5) (2005) 179-193. doi:10.1016/j.mbs.2005.02.004.

[12] M. A. El-Tawil, W. El-Tahan, A. Hussein, Using fem-rvt technique for solving a randomly excited ordinary differential equation with a random operator, Applied Mathematics and Computation 187 (2) (2007) 856-867. doi:10.16/j.amc.2006.08.164.

[13] M. C. Casabán, J. C. Cortés, J. V. Romero, M. D. Roselló, Determining the first probability density function of linear random initial value problems by the Random Variable Transformation (RVT) technique: A comprehensive study, Abstract and Applied Analysis 2014-ID248512 (2014) 1-25. doi:10.1155/2013/248512. 
[14] F. A. Dorini, M. S. Cecconello, L. B. Dorini, On the logistic equation subject to uncertainties in the environmental carrying capacity and initial population density, Communications in Nonlinear Science and Numerical Simulation 33 (2016) 160-173. doi:10.1016/j.cnsns.2014.12.016.

[15] M. C. Casabán, J. C. Cortés, A. Navarro-Quiles, J. V. Romero, M. D. Roselló, R. J. Villanueva, A comprehensive probabilistic solution of random SIS-type epidemiological models using the Random Variable Transformation technique, Communications in Nonlinear Science and Numerical Simulation 32 (2016) 199-210. doi:10.1016/j.cnsns.2014.12.016.

[16] A. Hussein, M. M. Selim, Solution of the stochastic transport equation of neutral particles with anisotropic scattering using RVT technique, Applied Mathematics and Computation 213 (1) (2009) $250-261$. doi:10.1016/j.amc.2009.03.016.

[17] A. Hussein, M. M. Selim, A developed solution of the stochastic Milne problem using probabilistic transformations, Applied Mathematics and Computation 216 (10) (2009) 2910-2919. doi:10.1016/j.amc.2010.04.003.

[18] A. Hussein, M. M. Selim, Solution of the stochastic radiative transfer equation with Rayleigh scattering using RVT technique, Applied Mathematics and Computation 218 (13) (2012) 7193-7203. doi:10.1016/j.amc.2011.12.088.

[19] L. T. Santos, F. A. Dorini, M. C. C. Cunha, The probability density function to the random linear transport equation, Applied Mathematics and Computation 216 (5) (2010) 1524-1530. doi:10.16/j.amc.2010.03.001.

[20] M. C. Casabán, J. C. Cortés, J. V. Romero, M. D. Roselló, Probabilistic solution of random homogeneous linear second-order difference equations, Applied Mathematics Letters 34 (2) (2014) $27-32$. doi:10.1016/j.aml.2014.03.0102.

[21] M. C. Casabán, J. C. Cortés, J. V. Romero, M. D. Roselló, Random first-order linear discrete models and their probabilistic solution: A comprehensive study, Abstract and Applied Analysis 2016-ID6372108 (2016) 1-22. doi:10.1155/2016/6372108.

[22] D. Y. Khusainov, M. Pokojovy, Solving the linear 1d thermoelasticity equations with pure delay, International Journal of Mathematics and Mathematical Siences 2015 (2015) 1-11. doi:10.1155/2015/479267.

[23] R. D. Driver, Ordinary and Delay Differential Equations, Springer-Verlag, New York, 1977. 